\title{
VARIATIONAL CONVERGENCE OVER METRIC SPACES
}

\author{
KAZUHIRO KUWAE AND TAKASHI SHIOYA
}

\begin{abstract}
We introduce a natural definition of $L^{p}$-convergence of maps, $p \geq 1$, in the case where the domain is a convergent sequence of measured metric space with respect to the measured Gromov-Hausdorff topology and the target is a Gromov-Hausdorff convergent sequence. With the $L^{p}$-convergence, we establish a theory of variational convergences. We prove that the Poincaré inequality with some additional condition implies the asymptotic compactness. The asymptotic compactness is equivalent to the Gromov-Hausdorff compactness of the energy-sublevel sets. Supposing that the targets are CAT(0)-spaces, we study convergence of resolvents. As applications, we investigate the approximating energy functional over a measured metric space and convergence of energy functionals with a lower bound of Ricci curvature.
\end{abstract}

\section{INTRODUCTION}

Let $M_{i} \rightarrow M$ and $Y_{i} \rightarrow Y(i=1,2,3, \ldots)$ be two pointed Gromov-Hausdorff convergent sequences of proper metric spaces, where 'proper' means that any bounded subset is relatively compact, and let us give measures on $M_{i}$ which converge to a measure on $M$. We are interested in the convergence and asymptotic behavior of maps $u_{i}: M_{i} \rightarrow Y_{i}$ and also energy functionals $E_{i}$ defined on a family of maps from $M_{i} \rightarrow Y_{i}$. Note that there are several attempts to define natural energy functionals on the mapping space from $M$ to $Y$ by the measured metric structure of $M$ and the metric structure of $Y$ (see, for example, $[9,30,4]$ for the case of $Y=\mathbb{R}$ and $[10,20,23,29]$ for the general $Y)$. We introduce a natural definition of the $L^{p}$-convergence of $u_{i}: M_{i} \rightarrow Y_{i}$ to $u: M \rightarrow Y, p \geq 1$, and establish a general theory for energy functionals $E_{i}$ by extending the theory of variational convergences, mainly studied by Mosco [27]. Mosco introduced the asymptotic compactness of energy functionals $\left\{E_{i}\right\}$, which is a generalization of the Rellich compactness. The asymptotic compactness is useful to obtain the convergence of energy minimizers, i.e., harmonic maps, and also to investigate spectral properties in the linear case. Under a uniform bound of Poincaré constants and some property of the metric on $M$, we prove the asymptotic compactness of $\left\{E_{i}\right\}$. We focus on a $\Gamma$-convergence with the asymptotic compactness, say compact convergence.

Received by the editors July 7, 2005.

2000 Mathematics Subject Classification. Primary 53C23; Secondary 49J45, 58E20.

Key words and phrases. Measured metric space, $L^{p}$-mapping space, Gromov-Hausdorff convergence, Mosco convergence, $\Gamma$-convergence, asymptotic compactness, the Poincaré inequality, CAT(0)-space, harmonic map, resolvent, spectrum.

The first author was partially supported by a Grant-in-Aid for Scientific Research No. 16540201 from the Ministry of Education, Science, Sports and Culture, Japan.

The second author was partially supported by a Grant-in-Aid for Scientific Research No. 14540056 from the Ministry of Education, Science, Sports and Culture, Japan. 
If $\left\{E_{i}\right\}$ is asymptotically compact, it has a compact convergent subsequence. We prove that the compact convergence is equivalent to the Gromov-Hausdorff convergence of the energy-sublevel sets, which is a geometric interpretation of compact convergence. We also prove that the compact convergence of $E_{i}$ is equivalent to a convergence of associated resolvents, provided that $Y_{i}$ are all CAT(0)-spaces and $E_{i}$ are lower semi-continuous convex functionals. Such a resolvent was defined by Jost [11] using the Moreau-Yosida approximation. As applications of our theory, we study the approximating energy functional and its spectral property. We also obtain the compactness of energy functionals if $M_{i}$ are Riemannian manifolds with a lower bound of Ricci curvature.

We mention further details. Let $M_{i} \rightarrow M$ and $Y_{i} \rightarrow Y$ be as above. For $p \geq 1$ and two measurable maps $u, v: M \rightarrow Y$, the $L^{p}$-distance $d_{L^{p}}(u, v)$ between $u$ and $v$ is defined by

$$
d_{L^{p}}(u, v):=\left(\int_{M} d_{Y}(u(x), v(x))^{p} d x\right)^{\frac{1}{p}} .
$$

Consider the $L^{p}$-metric space of measurable maps from $M$ to $Y$ (resp. from $M_{i}$ to $\left.Y_{i}\right)$ and denote it by $X\left(\operatorname{resp} . X_{i}\right)$. To define the $L^{p}$-convergence of maps $u_{i} \in X_{i}$ to a map $u \in X$, we take Gromov-Hausdorff approximations $\varphi_{i}: M_{i} \rightarrow M$, which are (not necessarily continuous) Borel maps almost preserving the distances. Assume first that $Y_{i}=Y$ for all $i$. It is natural to consider that $\varphi_{i} \circ u$ converges to $u$, so that if a sequence $u_{i}: M_{i} \rightarrow Y\left(=Y_{i}\right)$ satisfies $d_{L^{p}}\left(\varphi_{i} \circ u, u_{i}\right) \rightarrow 0$ as $i \rightarrow \infty$, then we say that $u_{i} L^{p}$-converges to $u$. However, this definition is natural only if $u$ is continuous, because the convergence of the measures on $M_{i}$ is only weak (see Remark 3.15 for the detailed explanation). If $Y_{i}=Y$ is (a subset of) a Banach space, then we can take a continuous $L^{p}$-approximation $\tilde{u}_{\epsilon}$ of a measurable $u: M \rightarrow Y, \epsilon>0$, such that $d_{L^{p}}\left(\tilde{u}_{\epsilon}, u\right)<\epsilon$. If

$$
\lim _{\epsilon \rightarrow 0} \varlimsup_{i \rightarrow \infty} d_{L^{p}}\left(\varphi_{i} \circ \tilde{u}_{\epsilon}, u_{i}\right)=0,
$$

then $u_{i} L^{p}$-converges to $u$. For general $Y_{i}, Y$, we embed all $Y_{i}$ and $Y$ into a Banach space and employ the same definition as above. This induces a topology on the disjoint union $\bigsqcup_{i} X_{i} \sqcup X$, say the $L^{p}$-topology. We prove that the $L^{p}$ topology is independent of the Gromov-Hausdorff approximation $\left\{\varphi_{i}\right\}$ and also of the embedding of $Y_{i}, Y$ into a Banach space. See Section 3.2 for the $L^{p}$-topology.

The $L^{p}$-topology on $\bigsqcup_{i} X_{i} \sqcup X$ has some nice properties involving the $L^{p}$-metric structure of $X_{i}$ and $X$, such as, if $X_{i} \ni u_{i}, v_{i} \rightarrow u, v \in X$ respectively in $L^{p}$, then $d_{L^{p}}\left(u_{i}, v_{i}\right) \rightarrow d_{L^{p}}(u, v)$. By their properties we present a set of axioms for a topology on $\bigsqcup_{i} X_{i} \sqcup X$ for general metric spaces $\left(X_{i}, d_{X_{i}}\right)$ and $\left(X, d_{X}\right)$. We call such a topology satisfying the axioms the asymptotic relation between $\left\{X_{i}\right\}$ and $X$ (see Definition 3.1). Since $X_{i}$ and $X$ are typically improper, the asymptotic relation can be thought as a nonuniform variant of Gromov-Hausdorff convergence $X_{i} \rightarrow X$.

After establishing the foundation of asymptotic relation, we give, in Section 4.1, a formulation of variational convergences of general functions $E_{i}: X_{i} \rightarrow[0,+\infty]$ to $E: X \rightarrow[0,+\infty] .\left\{E_{i}\right\}$ is said to be asymptotically compact if for any bounded sequence $u_{i} \in X_{i}$ with $\sup _{i} E_{i}\left(u_{i}\right)<+\infty$, it has a convergent subsequence, where 'bounded' means that $d_{X_{i}}\left(u_{i}, v_{i}\right)$ is bounded for some convergent sequence $v_{i} \in X_{i}$. We say that $E_{i} \Gamma$-converges to $E$ if the following $(\Gamma 1)$ and $(\Gamma 2)$ are both satisfied. 
(Г1) For any $u \in X$ there exists a sequence $u_{i} \in X_{i}$ such that $u_{i} \rightarrow u$ and $E_{i}\left(u_{i}\right) \rightarrow E(u)$.

(Г2) We have $\underline{\lim }_{i} E_{i}\left(u_{i}\right) \geq E(u)$ for any convergent sequence $X_{i} \ni u_{i} \rightarrow u \in X$.

We say that $E_{i}$ compactly converges to $E$ if $E_{i} \Gamma$-converges to $E$ and if $\left\{E_{i}\right\}$ is asymptotically compact.

In Section 4, we study the asymptotic compactness and the compact convergence of $\left\{E_{i}\right\}$. It is important to investigate under what condition the asymptotic compactness is obtained. We introduce a concept of the local covering order of a locally compact metric space (see Definition 4.13), which is a quantity measuring the local size of the metric space. Assume that $M_{i}$ and $M$ are all compact. Under a bound of a local covering order of $M$ and a uniform bound of Poincaré constants for $E_{i}$ on $M_{i}$, we prove the asymptotic compactness of $\left\{E_{i}\right\}$ (see Theorem 4.15). Since we do not need the doubling condition, our theorem can be applied to infinite-dimensional spaces. There is a mistake in the linear version in [22]. We correct it by introducing the local covering order.

Motivated by Gromov's study of spectral concentration, Section $3 \frac{1}{2} .57$ of [8], we prove that the compact convergence $E_{i} \rightarrow E$ is equivalent to the condition that for any $c \in \mathbb{R}$ there exists a sequence $c_{i} \searrow c \in \mathbb{R}$ such that the sublevel set $\left(\left\{u \in X_{i} \mid E_{i}(u) \leq c_{i}\right\}, o_{i}\right)$ converges to $(\{u \in X \mid E(u) \leq c\}, o)$ with respect to the pointed Gromov-Hausdorff topology, where $o_{i} \in X_{i}$ is a sequence converging to a point $o \in X$ (see Theorem 4.25).

In Section 5, we study variational convergences over CAT(0)-spaces, where a CAT(0)-space is a globally nonpositively curved metric space. Typical examples of CAT(0)-spaces are Hadamard manifolds and trees. If the target space is CAT $(0)$, then the $L^{2}$-mapping space is also $\operatorname{CAT}(0)$, and an energy functional defined in a suitable way becomes convex and lower semi-continuous. Thus, it is reasonable to assume that $X_{i}$ and $X$ are all CAT(0)-spaces, and $E_{i}$ and $E$ are convex lower semi-continuous functions with $E_{i}, E \not \equiv+\infty$. For any $\lambda \geq 0$ and $u \in X$, there exists a unique minimizer, say $J_{\lambda}^{E}(u) \in X$, of $v \mapsto \lambda E(v)+d_{X}(u, v)^{2}$. This defines a map $J_{\lambda}^{E}: X \rightarrow X$, called the resolvent of $E$ (see [11]). Note that if $X$ is a Hilbert space and if $E$ is a closed densely defined symmetric quadratic form on $X$, then we have $J_{\lambda}^{E}=(I+\lambda A)^{-1}$, where $A$ is the infinitesimal generator associated with $E$. The one-parameter family $[0,+\infty) \ni \lambda \mapsto J_{\lambda}^{E}(u)$ gives a deformation of a given map $u \in X$ to a minimizer of $E$ (or a harmonic map), $\lim _{\lambda \rightarrow+\infty} J_{\lambda}^{E}(u)$ (if any). Jost [14] studied convergence of resolvents and Moreau-Yosida approximations. Although his study is only on a fixed CAT(0)-space, we extend it for a sequence of CAT(0)spaces with an asymptotic relation. We investigate the relation between asymptotic compactness and resolvents (see Proposition 5.18). This is new even on a fixed CAT(0)-space. As the main theorem in this section (see Theorem 5.22), we prove that $E_{i}$ compactly converges to $E+c$ for some constant $c \in \mathbb{R}$ iff the following are both satisfied:

(1) For any $\lambda>0$ and for any bounded sequence $u_{i} \in X_{i}, J_{\lambda}^{E_{i}}\left(u_{i}\right)$ has a convergent subsequence.

(2) $J_{\lambda}^{E_{i}}\left(u_{i}\right)$ converges to $J_{\lambda}^{E}(u)$ for any $\lambda>0$ and for any convergent sequence $X_{i} \ni u_{i} \rightarrow u \in X$.

In the case where $E_{i}$ and $E$ are symmetric quadratic forms on Hilbert spaces, this theorem is obtained in our previous paper [22] (see also Section 5.4 of this paper) 
and is applied to the study of the spectral properties of the Laplacian of convergent Riemannian manifolds. This also has some applications to a homogenization problem, [31], and convergence of Dirichlet forms, [16, 17, 28], including finitedimensional approximation problems, $[19,18]$. The results of such studies could possibly extend to the nonlinear case.

In Section 6, we give some applications. Define the $\rho$-approximating energy $E^{\rho}(u), \rho>0$, of a measurable map $u: M \rightarrow Y$ by

$$
E^{\rho}(u):=\frac{1}{2} \int_{M} \frac{1}{|B(x, \rho)|} \int_{B(x, \rho) \backslash\{x\}} \frac{d_{Y}(u(x), u(y))^{p}}{\rho^{p}} d y d x \in[0,+\infty],
$$

where $|B(x, \rho)|$ is the measure of the open metric $\rho$-ball $B(x, \rho)$ centered at $x$. If $M$ and $Y$ are both complete Riemannian manifolds, then as $\rho \rightarrow 0+, E^{\rho} \Gamma$-converges to the usual energy functional up to a constant multiple over the $L^{p}$-mapping space. If $M$ is a Riemannian manifold and if $Y$ is a metric space, then $E^{\rho} \Gamma$-converges to some functional $E$, which is often called the Korevaar-Schoen energy functional (see [20]). We prove that $\left\{E^{\rho}\right\}$ is asymptotically compact, and so the convergence $E^{\rho} \rightarrow E$ becomes compact, provided $M$ is a compact Riemannian manifold and $Y$ is a proper metric space. More generally, this is true in the case where $M$ is a compact measured metric space with some property (called the measure contraction property) stated in $[30,23]$. Note that each $E^{\rho}$ does not have the Rellich compactness property. This brings an example of an asymptotically compact sequence $\left\{E_{i}\right\}$ with noncompact $E_{i}$. In the real-valued case (i.e., $Y=\mathbb{R}$ ), the functionals $E^{\rho}$ and $E$ are symmetric quadratic forms, and we denote their infinitesimal generators by $A^{\rho}$ and $A$ respectively. The operator $A$ is the Laplacian if $M$ is a Riemannian manifold. Each $A^{\rho}$ has a nonempty essential spectrum, whose bottom is divergent to $+\infty$ as $\rho \rightarrow 0+$. The $k^{t h}$ eigenvalue of $A^{\rho}$ converges to that of $A$ as $\rho \rightarrow 0+$ for any fixed $k \in \mathbb{N}$.

We next study the convergence of energy functionals under a lower bound of Ricci curvature. For a constant $n \geq 2$, let $M_{i}$ be a sequence of $n$-dimensional closed Riemannian manifolds of Ricci curvature $\geq-(n-1)$ which converges to a compact measured metric space $M$ with respect to the measured Gromov-Hausdorff topology. Let $Y_{i} \rightarrow Y$ be a pointed Gromov-Hausdorff convergent sequence of proper pointed metric spaces. Consider the Korevaar-Schoen type energy, say $E_{i}$, on the $L^{2}$-mapping space from $M_{i}$ to $Y_{i}$ (see $[20]$ ). We prove that $\left\{E_{i}\right\}$ is asymptotically compact and has a compactly convergent subsequence (see Theorem 6.6). If $Y_{i}=Y$ is a fixed complete Riemannian manifold, then Kasue's result [16], together with that of Cheeger-Colding [5], shows that $E_{i}$ converges in some sense to a naturally defined energy functional $E$ on the $L^{2}$-mapping space from $M$ to $Y$. Combining this with our result yields that $E_{i}$ compactly converges to $E$.

\section{Preliminaries for Gromov-Hausdorff Convergence}

The Hausdorff distance $d_{H}^{Z}(X, Y)$ between two subsets $X$ and $Y$ of a metric space $Z$ is defined to be the infimum of $r>0$ such that $X \subset B(Y, r)$ and $Y \subset B(X, r)$, where $B(X, r)$ denotes the open $r$-neighborhood of $X$. Let $X$ and $Y$ be two compact metric spaces. The Gromov-Hausdorff distance $d_{G H}(X, Y)$ between $X$ and $Y$ is the infimum of $d_{H}^{Z}(X, Y)$, where $Z$ is any metric space into which $X$ and $Y$ are isometrically embedded. The distortion $\operatorname{dis} \varphi$ of a (not necessarily continuous) map 
$\varphi: X \rightarrow Y$ is defined by

$$
\operatorname{dis} \varphi:=\sup _{x, y \in X}|d(\varphi(x), \varphi(y))-d(x, y)|,
$$

where $d$ denotes the distance function. If a map $\varphi: X \rightarrow Y$ satisfies $\operatorname{dis} \varphi<\epsilon$ and $B(\varphi(X), \epsilon)=Y$, then it is called an $\epsilon$-approximation. It is known that if $d_{G H}(X, Y)<\epsilon$, then there exists a $2 \epsilon$-approximation from $X$ to $Y$. Conversely, if there exists an $\epsilon$-approximation from $X$ to $Y$, then $d_{G H}(X, Y)<2 \epsilon$.

Let $\{i\}$ be a directed set and $\left\{\left(X_{i}, o_{i}\right)\right\}$ a net of pointed proper metric spaces, where 'proper' means that any closed and bounded subset is compact. We say that $\left(X_{i}, o_{i}\right)$ converges to a pointed proper metric space $(X, o)$ with respect to the pointed Gromov-Hausdorff convergence if for any $r>0$ there exist two nets of positive numbers $r_{i} \searrow r, \epsilon_{i} \rightarrow 0+$, and $\epsilon_{i}$-approximations $\varphi_{i}: B\left(o_{i}, r_{i}\right) \rightarrow B(o, r)$ with $\varphi_{i}\left(o_{i}\right)=o$. This is equivalent to the existence of $\epsilon_{i}$-approximations $\psi_{i}: B\left(o_{i}, r_{i}^{\prime}\right) \rightarrow$ $B\left(o, r_{i}\right)$ such that $\psi_{i}\left(o_{i}\right)=o, \epsilon_{i} \rightarrow 0+$ and $r_{i}^{\prime}>r_{i} \rightarrow+\infty$. We call such a $\left\{\psi_{i}\right\}$ a pointed Gromov-Hausdorff approximation. This convergence induces a topology, called the pointed Gromov-Hausdorff topology, on the set of pointed proper metric spaces.

Note that a notion of convergence of a sequence of countable elements is not enough to define a topology, and we need convergence of a net indexed by a directed set for it.

The following definition seems not to be common knowledge.

Definition 2.1 (Compact Hausdorff convergence). Let $A_{i}, A$ be closed subsets of a metric space $Z$. We say that $A_{i}$ converges to $A$ in the compact Hausdorff convergence if for some point $o \in Z$ and for any $r>0$ there exists a net $r_{i} \searrow r$ such that

$$
d_{H}^{Z}\left(A_{i} \cap B\left(o, r_{i}\right), A \cap B(o, r)\right) \rightarrow 0 .
$$

Remark 2.2. If the restrictions of the metric on $A_{i}$ and $A$ are all proper, then a compact Hausdorff convergence $A_{i} \rightarrow A$ implies the pointed Gromov-Hausdorff convergence $\left(A_{i}, o_{i}\right) \rightarrow(A, o)$ for any net $o_{i} \in X$ converging to a point $o \in X$.

The following proposition seems to be well-known.

Proposition 2.3 (cf. Proof of $3.5(\mathrm{~b})$ of $[8]$ ). Let $\left(X_{i}, o_{i}\right)$ be a net of pointed proper metric spaces converging to a pointed proper metric space $(X, o)$ in the pointed Gromov-Hausdorff topology. Then, there exists a proper metric $d_{\mathcal{X}}$ on the disjoint union $\mathcal{X}:=\bigsqcup_{i} X_{i} \sqcup X$ such that

(1) the restrictions of $d_{\mathcal{X}}$ on $X_{i}$ and $X$ coincide with the original metrics $d_{X_{i}}$ and $d_{X}$ respectively;

(2) $X_{i}$ converges to $X$ in the compact Hausdorff convergence in $\left(\mathcal{X}, d_{\mathcal{X}}\right)$;

(3) $d_{\mathcal{X}}\left(o_{i}, o\right) \rightarrow 0$.

Proof. Since the proof is standard, here we give an outline. Take a dense countable subset $\left\{p_{n}\right\}_{n \in \mathbb{N}} \subset X$ with $p_{1}=o$. By the convergence $\left(X_{i}, o_{i}\right) \rightarrow(X, o)$, there exist a net $N(i) \nearrow \infty$ of natural numbers and points $p_{n, i} \in X_{i}$ for $n=1,2, \ldots, N(i)$ with $p_{1, i}=o_{i}$ such that

$$
\left|d_{X_{i}}\left(p_{m, i}, p_{n, i}\right)-d_{X}\left(p_{m}, p_{n}\right)\right|<1 / N(i)
$$


for any $m, n=1,2, \ldots, N(i)$. Set $d_{\mathcal{X}}:=d_{X_{i}}$ on $X_{i} \times X_{i}$ and $d_{\mathcal{X}}:=d_{X}$ on $X \times X$. We define the distance $d_{\mathcal{X}}(x, y)$ between any two points $x \in X_{i}$ and $y \in X$ by

$$
d_{\mathcal{X}}(x, y):=\inf _{n=1,2, \ldots, N(i)} d_{X_{i}}\left(x, p_{n, i}\right)+d_{X}\left(y, p_{n}\right)+1 / N(i)
$$

and the distance $d_{\mathcal{X}}(x, y)$ between $x \in X_{i}$ and $y \in X_{j}$ for $i \neq j$ by

$$
d_{\mathcal{X}}(x, y):=\inf _{z \in X} d_{\mathcal{X}}(x, z)+d_{\mathcal{X}}(y, z) .
$$

Then, $d_{\mathcal{X}}$ is a unique minimal distance function on $\mathcal{X}$ satisfying (1) and the condition that $d_{\mathcal{X}}\left(p_{n, i}, p_{n}\right)=1 / N(i)$ for all $n=1,2, \ldots, N(i)$. In particular we have (3). It is easy to verify (2). This completes the proof.

We assume that all measure spaces are locally compact Polish spaces with positive Radon measures of full support, where a Polish space is, by definition, homeomorphic to a complete separable metric space.

Definition 2.4 (Measure approximation, [22]). Let $M_{i}$ and $M$ be measure spaces. A net $\left\{\varphi_{i}: M_{i} \supset \mathcal{D}\left(\varphi_{i}\right) \rightarrow M\right\}$ of maps is called a measure approximation if the following are satisfied:

(M1) Each $\varphi_{i}$ is a measurable map from a Borel subset $\mathcal{D}\left(\varphi_{i}\right)$ of $M_{i}$ to $M$.

(M2) The push-forward by $\varphi_{i}$ of the measure on $M_{i}$ vaguely (or weakly-star) converges to the measure on $M$, i.e., for any $f \in C_{0}(M)$,

$$
\lim _{i} \int_{\mathcal{D}\left(\varphi_{i}\right)} f \circ \varphi_{i}(x) d x=\int_{M} f(x) d x
$$

where $C_{0}(M)$ is the set of continuous functions on $M$ with compact support.

Note that it is nonsense to define a topology on the set of measure spaces by the existence of a measure approximation. This is because, if the total measure of $M_{i}$ converges to a finite number $a$, then the map from $M_{i}$ to the set of a single point with mass $a$ forms a measure approximation. However, the following definition makes sense.

Definition 2.5 (Measured Gromov-Hausdorff topology, $[7,8]$ ). Let $M_{i}$ and $M$ be compact measured metric spaces. We say that $M_{i}$ converges to $M$ in the sense of the measured Gromov-Hausdorff convergence if there exists a measure approximation $\left\{\varphi_{i}: M_{i} \rightarrow M\right\}$ such that each $\varphi_{i}$ is an $\epsilon_{i}$-approximation for some $\epsilon_{i} \rightarrow 0+$.

The pointed version is also defined. Let $M_{i}$ and $M$ be pointed proper measured metric spaces. We say that $M_{i}$ converges to $M$ in the sense of the pointed measured Gromov-Hausdorff convergence if there exists a pointed Gromov-Hausdorff approximation that is a measure approximation, which we call a measured pointed Gromov-Hausdorff approximation.

The (pointed) measured Gromov-Hausdorff convergence induces a topology, called the (pointed) measured Gromov-Hausdorff topology, on the set of compact (resp. pointed proper) measured metric spaces, which is stronger than the (pointed) Gromov-Hausdorff topology.

\section{Asymptotic Relation}

In Section 3.1, we present a set of axioms of asymptotic relation and prove some lemmas needed in the later sections. Then, in Section 3.2, we prove that $L^{p_{-}}$ mapping spaces $X_{i}$ and $X$ as in the Introduction satisfy the axioms. The axioms are more flexible than our previous ones in the linear case, [22]. 
3.1. Foundation. Throughout this paper, we denote by $i$ any element of a given directed set $\{i\}$. Let $\left\{X_{i}\right\}$ be a net of metric spaces and $X$ a metric space. Define

$$
\mathcal{X}:=\left(\bigsqcup_{i} X_{i}\right) \sqcup X \quad \text { (disjoint union). }
$$

We sometimes consider the following additional condition:

(L) Given a field $K=\mathbb{R}$ or $\mathbb{C}, X_{i}$ and $X$ are all topological linear spaces over $K$ whose topologies are compatible with their metric structure.

Definition 3.1 (Asymptotic relation). We call a topology on $\mathcal{X}$ satisfying the following an asymptotic relation between $\left\{X_{i}\right\}$ and $X$ :

(A1) $X_{i}$ and $X$ are all closed in $\mathcal{X}$, and the restricted topology of $\mathcal{X}$ on each of $X_{i}$ and $X$ coincides with its original topology.

(A2) For any $x \in X$ there exists a net $x_{i} \in X_{i}$ converging to $x$ in $\mathcal{X}$.

(A3) If $X_{i} \ni x_{i} \rightarrow x \in X$ and $X_{i} \ni y_{i} \rightarrow y \in X$ in $\mathcal{X}$, then we have $d_{X_{i}}\left(x_{i}, y_{i}\right) \rightarrow$ $d_{X}(x, y)$.

(A4) If $X_{i} \ni x_{i} \rightarrow x \in X$ in $\mathcal{X}$ and if $y_{i} \in X_{i}$ is a net with $d_{X_{i}}\left(x_{i}, y_{i}\right) \rightarrow 0$, then $y_{i} \rightarrow x$ in $\mathcal{X}$.

Supposing (L), we say that an asymptotic relation between $\left\{X_{i}\right\}$ and $X$ is linear if the following is satisfied:

(AL) If $X_{i} \ni x_{i} \rightarrow x \in X$ and $X_{i} \ni y_{i} \rightarrow y \in X$ in $\mathcal{X}$, then $a x_{i}+b y_{i} \rightarrow a x+b y$ in $\mathcal{X}$ for any scalers $a, b \in K$.

By the definition, an asymptotic relation on $\mathcal{X}$ is a Hausdorff topology.

Remark 3.2. Notice that a Gromov-Hausdorff convergence $X_{i} \rightarrow X$ induces an asymptotic relation (see Proposition 3.5(b) of [8] and also Proposition 2.3 of this paper). Thus, the existence of an asymptotic relation between $\left\{X_{i}\right\}$ and $X$ can be thought of as a generalization of Gromov-Hausdorff convergence $X_{i} \rightarrow X$ in a sense. However, it seems not to be suitable to call an asymptotic relation a convergence. This is because the existence of an asymptotic relation between $\left\{X_{i}\right\}$ and $X$ induces one between $\left\{X_{i}\right\}$ and any $Y \subset X$ as the restriction.

Definition 3.3 (Metric approximation). A net $\left\{f_{i}: X \supset \mathcal{D}\left(f_{i}\right) \rightarrow X_{i}\right\}$ of (not necessarily continuous) maps is called a metric approximation for $\left\{X_{i}\right\}$ and $X$ if the following are satisfied:

(B1) $\mathcal{D}\left(f_{i}\right)$ is monotone nondecreasing in $i$ and $\bigcup_{i} \mathcal{D}\left(f_{i}\right)$ is dense in $X$.

(B2) For any $x, y \in \bigcup_{i} \mathcal{D}\left(f_{i}\right)$ we have $d_{X_{i}}\left(f_{i}(x), f_{i}(y)\right) \rightarrow d_{X}(x, y)$.

Under $(\mathrm{L})$, a metric approximation $\left\{f_{i}: X \supset \mathcal{D}\left(f_{i}\right) \rightarrow X_{i}\right\}$ is said to be linear if the following is satisfied:

(BL) Each $f_{i}$ is a linear map from a linear subspace $\mathcal{D}\left(f_{i}\right) \subset X$ to $X_{i}$.

The concept of metric approximation is needed to define $L^{p}$-topology in Section 3.2 .

Definition 3.4 (Compatibility). For an asymptotic relation and a metric approximation $\left\{f_{i}\right\}$ between $\left\{X_{i}\right\}$ and $X$, we consider the following compatibility condition between them:

(C) $f_{i}(x) \rightarrow x$ in $\mathcal{X}$ for any $x \in \bigcup_{i} \mathcal{D}\left(f_{i}\right)$. 
If the compatibility condition holds, we say that the asymptotic relation and the metric approximation $\left\{f_{i}\right\}$ are compatible to each other.

Lemma 3.5. (1) For a given (linear) asymptotic relation between $\left\{X_{i}\right\}$ and $X$, there exists a (linear) metric approximation $\left\{f_{i}\right\}$ compatible with it such that $\mathcal{D}\left(f_{i}\right)=X$ for all $i$ in the nonlinear case and that $\mathcal{D}\left(f_{i}\right)$ consists of finite linear combinations of a given complete basis on $X$ in the linear case.

(2) For a given (linear) metric approximation $\left\{f_{i}\right\}$ for $\left\{X_{i}\right\}$ and $X$, there exists a unique (linear) asymptotic relation between $\left\{X_{i}\right\}$ and $X$ compatible with $\left\{f_{i}\right\}$.

Proof. (1): Let an asymptotic relation between $\left\{X_{i}\right\}$ and $X$ be given. By (A2), for each $x \in X$ we can choose a net $x_{i} \in X_{i}$ such that $x_{i} \rightarrow x$ with respect to the asymptotic relation and set $f_{i}(x):=x_{i}$. This defines a map $f_{i}: \mathcal{D}\left(f_{i}\right)=X \rightarrow X_{i}$. (A3) implies (B2).

Assume that the asymptotic relation is linear and let $\mathcal{B}$ be a complete linear basis on $X$. We define a map $f_{i}$ on $\mathcal{B}$ in the same way as above. Then it extends to a linear map from the set of finite linear combinations of $\mathcal{B}$, say $\mathcal{D}\left(f_{i}\right)$, to $X_{i}$. $\mathcal{D}\left(f_{i}\right)$ is a dense linear subspace of $X$.

(2): Let $\left\{f_{i}\right\}$ be a metric approximation for $\left\{X_{i}\right\}$ and $X$. We define a convergence $X_{i} \ni x_{i} \rightarrow x \in X$ by the following condition: There exists a sequence $\tilde{x}_{j} \in \bigcup_{i} \mathcal{D}\left(f_{i}\right)$, $j=1,2, \ldots$, converging to $x$ in $X$ such that

$$
\lim _{j} \varlimsup_{i} d_{X_{i}}\left(x_{i}, f_{i}\left(\tilde{x}_{j}\right)\right)=0
$$

(compare (1.1)). This convergence together with (A1) induces a unique topology on $\mathcal{X}$. We shall show that this topology is an asymptotic relation. In fact, (B1) implies (A2). Let us verify (A3). Assume $X_{i} \ni x_{i} \rightarrow x \in X$ and $X_{i} \ni y_{i} \rightarrow y \in X$. Then there exist $\tilde{x}_{j}, \tilde{y}_{j} \in \bigcup_{i} \mathcal{D}\left(f_{i}\right)$ such that

$$
\begin{aligned}
& \tilde{x}_{j} \rightarrow x, \quad \lim _{j} \varlimsup_{i} d_{X_{i}}\left(x_{i}, f_{i}\left(\tilde{x}_{j}\right)\right)=0, \\
& \tilde{y}_{j} \rightarrow y, \quad \lim _{j} \varlimsup_{i} d_{X_{i}}\left(y_{i}, f_{i}\left(\tilde{y}_{j}\right)\right)=0 .
\end{aligned}
$$

Since $\left\{f_{i}\right\}$ is a metric approximation, we have

$$
\lim _{j} \lim _{i} d_{X_{i}}\left(f_{i}\left(\tilde{x}_{j}\right), f_{i}\left(\tilde{y}_{j}\right)\right)=\lim _{j} d_{X}\left(\tilde{x}_{j}, \tilde{y}_{j}\right)=d_{X}(x, y) .
$$

Therefore the triangle inequalities show that $d_{X_{i}}\left(x_{i}, y_{i}\right) \rightarrow d_{X}(x, y)$. (A4) is obtained from the definition of the convergence and a triangle inequality. Thus the topology on $\mathcal{X}$ defined here is an asymptotic relation. It is obvious that the compatibility condition is satisfied.

Supposing that $\left\{f_{i}\right\}$ is linear, it is easy to see that the asymptotic relation defined above is linear.

Definition 3.6 (Asymptotic continuity). Let $\left\{X_{i}\right\}$ and $X$ have an asymptotic relation. A compatible metric approximation $\left\{f_{i}\right\}$ is said to be asymptotically continuous if $\mathcal{D}\left(f_{i}\right)=X$ for any $i$ and if $f_{i}\left(x_{i}\right) \rightarrow x$ in $\mathcal{X}$ holds for any net $X \ni x_{i} \rightarrow x \in X$.

Lemma 3.7. Assume that $\left\{X_{i}\right\}$ and $X$ have a (linear) asymptotic relation and that $X$ is separable. Then, there exists an asymptotically continuous (linear) metric approximation $\left\{f_{i}: \mathcal{D}\left(f_{i}\right)=X \rightarrow X_{i}\right\}$ compatible with it such that each $f_{i}$ is a Borel map. 
Proof. Let us first consider the nonlinear case. Take a dense countable subset $\left\{a_{k}\right\}_{k \in \mathbb{N}}$ of $X$ and set $A_{n}:=\left\{a_{1}, a_{2}, \ldots, a_{n}\right\}$. For a given $x \in X$ we choose $a_{k}$ with smallest $k$ among the points in $A_{n}$ nearest to $x$; then we set $\pi_{n}(x):=a_{k}$. This defines a Borel map $\pi_{n}: X \rightarrow A_{n}$. By Lemma 3.5, there exists a metric approximation $\left\{g_{i}: X \rightarrow X_{i}\right\}$ compatible with the given asymptotic relation between $\left\{X_{i}\right\}$ and $X$. Let

$$
\epsilon_{n, i}:=\sup \left\{\left|d_{X_{j}}\left(g_{j}(a), g_{j}\left(a^{\prime}\right)\right)-d_{X}\left(a, a^{\prime}\right)\right| \mid j \geq i, a, a^{\prime} \in A_{n}\right\} .
$$

Then, for each $n \in \mathbb{N}$ we have $\lim _{i} \epsilon_{n, i}=0$. Hence, there exists a net $n(i) \rightarrow \infty$ such that $\lim _{i} \epsilon_{n(i), i}=0$. Define $f_{i}:=g_{i} \circ \pi_{n(i)}$. Since the image of each $\pi_{n(i)}$ consists of finitely many points, it is a Borel map. We take any $x, x^{\prime} \in X$ and fix them. It then follows that

$$
\begin{aligned}
\mid d_{X_{i}}\left(f_{i}(x), f_{i}\left(x^{\prime}\right)\right)- & d_{X}\left(x, x^{\prime}\right) \mid \\
\leq & \left|d_{X_{i}}\left(f_{i}(x), f_{i}\left(x^{\prime}\right)\right)-d_{X}\left(\pi_{n(i)}(x), \pi_{n(i)}\left(x^{\prime}\right)\right)\right| \\
& +\left|d_{X}\left(\pi_{n(i)}(x), \pi_{n(i)}\left(x^{\prime}\right)\right)-d_{X}\left(x, x^{\prime}\right)\right| \\
\leq & \epsilon_{n(i), i}+d_{X}\left(\pi_{n(i)}(x), x\right)+d_{X}\left(\pi_{n(i)}\left(x^{\prime}\right), x^{\prime}\right) \\
\rightarrow & 0,
\end{aligned}
$$

so that $\left\{f_{i}\right\}$ is a metric approximation. The rest of the proof is to show the asymptotic continuity of $\left\{f_{i}\right\}$. Take points $x_{i}, x \in X$ such that $x_{i} \rightarrow x$. Since $\pi_{n(i)}\left(x_{i}\right)$ and $\pi_{n(i)}(x)$ both tend to $x$, we have $d_{X}\left(\pi_{n(i)}\left(x_{i}\right), \pi_{n(i)}(x)\right) \rightarrow 0$, which together with $\epsilon_{n(i), i} \rightarrow 0$ implies $d_{X}\left(f_{i}\left(x_{i}\right), f_{i}(x)\right) \rightarrow 0$. By (A4), we obtain $f_{i}\left(x_{i}\right) \rightarrow x$ in $\mathcal{X}$. This completes the proof in the nonlinear case.

Assume that the asymptotic relation is linear. Since $X$ is separable, there is a countable complete linear basis $\mathcal{B}=\left\{a_{1}, a_{2}, \ldots\right\}$ of $X$ such that $d_{X}\left(o, a_{k}\right)$ is bounded away from zero and from infinity as $k \rightarrow \infty$. We have a unique Hilbert metric on $X$ for which $\mathcal{B}$ is a complete orthonormal basis. It follows that the topology of the Hilbert metric on $X$ coincides with that of $d_{X}$. Let $A_{n}$ be the linear subspace spanned by $a_{1}, a_{2}, \ldots, a_{n}$ and $\pi_{n}: X \rightarrow A_{n}$ the orthogonal projection. Every $\pi_{n}$ is continuous and satisfies that $\pi_{m}=\pi_{m} \circ \pi_{n}$ for $n \leq m$. By Lemma 3.5, we find a linear metric approximation $\left\{g_{i}\right\}$ compatible with the asymptotic relation such that $\mathcal{D}\left(g_{i}\right)$ consists of finite linear combinations of $\mathcal{B}$. Let

$$
\epsilon_{n, i}:=\sup \left\{\left|d_{X_{j}}\left(g_{j}(a), g_{j}\left(a^{\prime}\right)\right)-d_{X}\left(a, a^{\prime}\right)\right| \mid j \geq i, a, a^{\prime} \in A_{n} \cap B(o, 1 / n)\right\},
$$

where $o \in X$ is the origin. The rest of the proof is the same as in the nonlinear case above.

Lemma 3.8. Assume that $\left\{X_{i}\right\}$ and $X$ have an asymptotic relation and $\left\{f_{i}\right\}$ an asymptotically continuous metric approximation compatible with it. Then, for any compact subset $C \subset X$ we have $\left.\operatorname{dis} f_{i}\right|_{C} \rightarrow 0$.

Proof. If not, there exist a number $\delta>0$ and nets $x_{i}, y_{i} \in C$ such that

$$
\left|d_{X_{i}}\left(f_{i}\left(x_{i}\right), f_{i}\left(y_{i}\right)\right)-d_{X}\left(x_{i}, y_{i}\right)\right| \geq \delta .
$$

Since $C$ is compact, by replacing the nets by subnets, we may assume that $x_{i} \rightarrow x$, $y_{i} \rightarrow y$ for some points $x, y \in C$. Then we have $d_{X}\left(x_{i}, y_{i}\right) \rightarrow d_{X}(x, y)$ and, by the asymptotic continuity of $\left\{f_{i}\right\}$,

$$
d_{X_{i}}\left(f_{i}\left(x_{i}\right), f_{i}(x)\right) \rightarrow 0, \quad d_{X_{i}}\left(f_{i}\left(y_{i}\right), f_{i}(y)\right) \rightarrow 0 .
$$


Moreover, $d_{X_{i}}\left(f_{i}(x), f_{i}(y)\right) \rightarrow d_{X}(x, y)$. Thus, by using triangle inequalities, the left-hand side of (3.1) tends to zero. This is a contradiction.

We shall briefly mention a connection between asymptotic relation and GromovHausdorff convergence.

Definition 3.9 (Asymptotic compactness). Assume that $\left\{X_{i}\right\}$ and $X$ have an asymptotic relation. We say that a net $x_{i} \in X_{i}$ is bounded if $d_{X_{i}}\left(x_{i}, o_{i}\right)$ is bounded for some convergent net $o_{i} \in X_{i}$. The asymptotic relation is said to be asymptotically compact if any bounded net $x_{i} \in X_{i}$ has a convergent subnet in $\mathcal{X}$ with respect to the asymptotic relation.

Proposition 3.10. If there exists an asymptotically compact asymptotic relation between $\left\{X_{i}\right\}$ and $X$, then $X$ is proper.

Proposition 3.11. Let $X_{i}$ and $X$ be proper metric spaces, let $o_{i} \in X_{i}$ be a net, and let $o \in X$ be a point. Then, the pointed space $\left(X_{i}, o_{i}\right)$ converges to $(X, o)$ in the Gromov-Hausdorff topology iff there exists an asymptotically compact asymptotic relation between $\left\{X_{i}\right\}$ and $X$ for which $o_{i}$ converges to o.

The proofs of Propositions 3.10 and 3.11 are easy. Also, they both follow from Theorem 4.25 below by setting $E_{i}:=0$ and $E:=0$.

3.2. Asymptotic relation between $L^{p}$-spaces. Let $M$ be a measure space (as we said in Section 2, it is a locally compact Polish space with a full supported Radon measure), $Y$ a metric space, and $p \geq 1$ a real number. Given two measurable maps $u, v: M \rightarrow Y$, we define the $L^{p}$-distance $d_{L^{p}}(u, v)$ between them by

$$
d_{L^{p}}(u, v):=\left(\int_{M} d_{Y}(u(x), v(x))^{p} d x\right)^{\frac{1}{p}} \leq+\infty,
$$

where $\int_{M} d x$ means integrating over $M$ by the measure on $M$. For a measurable map $\xi: M \rightarrow Y$ we define

$$
L_{\xi}^{p}(M, Y):=\left\{u: M \rightarrow Y \mid \text { measurable map with } d_{L^{p}}(u, \xi)<+\infty\right\} .
$$

We identify two maps in $L_{\xi}^{p}(M, Y)$ if they are equal a.e. on $M$, so that $L_{\xi}^{p}(M, Y)$ becomes a metric space with metric $d_{L^{p}}$. If $Y$ is complete (resp. separable), then $L_{\xi}^{p}(M, Y)$ is also complete (resp. separable).

We first introduce a natural asymptotic relation between $L^{p}$-spaces in the case where the target space is a Banach space. Let $\mathbb{B}$ be a Banach space over $\mathbb{R}$ with norm $\|\cdot\|$ and origin $o$. Note that $L_{o}^{p}(M, \mathbb{B})$ is a Banach space with respect to $d_{L^{p}}$. The support 'supp $u$ ' of a measurable map $u: M \rightarrow Y$ is defined to be the subset of $M$ satisfying the condition that $x \in M \backslash \operatorname{supp} u$ iff there exists an open neighborhood $U$ of $x$ such that $u=o$ a.e. on $U$. Denote by $C_{o}(M, \mathbb{B})$ the set of continuous maps $u: M \rightarrow \mathbb{B}$ with compact support supp $u$. We have the following lemma in a standard way, and the proof is omitted.

Lemma 3.12. $C_{o}(M, \mathbb{B})$ is a dense linear subspace of $L_{o}^{p}(M, \mathbb{B})$.

Let $\left\{\varphi_{i}: M_{i} \supset \mathcal{D}\left(\varphi_{i}\right) \rightarrow M\right\}$ be a measure approximation for measure spaces $M_{i}$ and $M$. For $u \in C_{o}(M, \mathbb{B})$, we define

$$
\Phi_{i} u(x):= \begin{cases}u \circ \varphi_{i}(x) & \text { for } x \in \mathcal{D}\left(\varphi_{i}\right), \\ o & \text { for } x \in M_{i} \backslash \mathcal{D}\left(\varphi_{i}\right) .\end{cases}
$$


For each $i, \Phi_{i} u: M_{i} \rightarrow \mathbb{B}$ is a measurable map and $\Phi_{i}$ is a linear map from $C_{o}(M, \mathbb{B})$. Define $\mathcal{D}\left(\Phi_{i}\right)$ to be the set of $u \in C_{o}(M, \mathbb{B})$ such that $d_{L^{p}}\left(\Phi_{j} u, o\right)<+\infty$ for any $j \geq i$. It is easy to prove that $\mathcal{D}\left(\Phi_{i}\right)$ is a linear subspace of $C_{o}(M, \mathbb{B})$.

Proposition 3.13. We have

(1) $\bigcup_{i} \mathcal{D}\left(\Phi_{i}\right)=C_{o}(M, \mathbb{B})$;

(2) $\left\{\Phi_{i}: L_{o}^{p}(M, \mathbb{B}) \supset \mathcal{D}\left(\Phi_{i}\right) \rightarrow L_{o}^{p}\left(M_{i}, \mathbb{B}\right)\right\}$ is a linear metric approximation.

Proof. We take any two maps $u, v \in C_{o}(M, \mathbb{B})$ and fix them. Since $M \ni x \mapsto$ $\|u(x)-v(x)\|^{p}$ is a continuous function with compact support, it follows from Definition 2.4(M2) that

$$
\begin{aligned}
d_{L^{p}}\left(\Phi_{i} u, \Phi_{i} v\right)^{p} & =\int_{\mathcal{D}\left(\varphi_{i}\right)}\left\|u \circ \varphi_{i}(x)-v \circ \varphi_{i}(x)\right\|^{p} d x \\
& \rightarrow \int_{M}\|u(x)-v(x)\|^{p} d x=d_{L^{p}}(u, v)^{p} .
\end{aligned}
$$

In particular,

$$
\lim _{i} d_{L^{p}}\left(\Phi_{i} u, o\right)=\lim _{i} d_{L^{p}}\left(\Phi_{i} u, \Phi_{i} o\right)=d_{L^{p}}(u, o)<+\infty,
$$

which implies $(1)$. By recalling that $C_{o}(M, \mathbb{B})$ is dense in $L_{o}^{p}(M, \mathbb{B})$, we obtain $(2)$. This completes the proof.

Definition 3.14 ( $L^{p}$-topology). As is seen in Lemma 3.5, the metric approximation $\left\{\Phi_{i}\right\}$ induces a unique linear asymptotic relation between $X_{i}:=L_{o}^{p}\left(M_{i}, \mathbb{B}\right)$ and $X:=L_{o}^{p}(M, \mathbb{B})$. We call the topology on $\mathcal{X}=\left(\bigsqcup_{i} X_{i}\right) \sqcup X$ the $L^{p}$-topology and a convergence for it an $L^{p}$-convergence.

Remark 3.15. In the proof of Proposition 3.13, the continuity of the maps $u$ and $v$ is necessary, so that the dense property of $C_{o}(M, \mathbb{B})$ in $L_{o}^{p}(M, \mathbb{B})$ is important to define the $L^{p}$-topology. If the target $Y$ is a general metric space, then $C_{o}(M, Y)$ is not necessarily dense in $L_{o}^{p}(M, Y)$ (e.g. in the case where $Y$ is disconnected).

We next consider the case where the targets are spaces of a Gromov-Hausdorff convergent net. Let $\left(Y_{i}, y_{i}\right)$ and $\left(Y, y_{0}\right)$ be pointed proper metric spaces such that $\left(Y_{i}, y_{i}\right)$ converges to $\left(Y, y_{0}\right)$ in the pointed Gromov-Hausdorff topology.

Lemma 3.16. There exists a separable real Banach space $(\mathbb{B},\|\cdot\|)$ into which all $Y_{i}$ and $Y$ are embedded isometrically.

Proof. By Proposition 2.3, all $Y_{i}$ and $Y$ can be isometrically embedded into the metric space $\mathcal{Y}:=\left(\bigsqcup_{i} Y_{i}\right) \sqcup Y$ with metric $d \mathcal{Y}$. As is well-known, we can isometrically embed $\mathcal{Y}$ into the real Banach space, say $(\mathbb{B},\|\cdot\|)$, consisting of continuous bounded functions on $\mathcal{Y}$ with uniform norm. The embedding map is

$$
\mathcal{Y} \ni y \mapsto d_{\mathcal{Y}}(y, \cdot)-d_{\mathcal{Y}}\left(y_{0}, \cdot\right) \in \mathbb{B} .
$$

Since $\mathcal{Y}$ is separable, so is $\mathbb{B}$.

We take $(\mathbb{B},\|\cdot\|)$ as in the lemma. Let $\xi_{i}: M_{i} \rightarrow Y_{i} \subset \mathbb{B}$ be measurable maps and $\xi: M \rightarrow Y \subset \mathbb{B}$ a continuous map such that

$$
\lim _{i} \int_{\mathcal{D}\left(\varphi_{i}\right)}\left\|\xi_{i}(x)-\xi \circ \varphi_{i}(x)\right\|^{p} d x=0
$$


and consider $L_{\xi_{i}}^{p}\left(M_{i}, Y_{i}\right)$ and $L_{\xi}^{p}(M, Y)$. We embed them to $L_{o}^{p}\left(M_{i}, \mathbb{B}\right)$ and $L_{o}^{p}(M, \mathbb{B})$ respectively by

$$
\begin{gathered}
\iota_{i}: L_{\xi_{i}}^{p}\left(M_{i}, Y_{i}\right) \ni u \mapsto u-\xi_{i} \in L_{o}^{p}\left(M_{i}, \mathbb{B}\right), \\
\iota: L_{\xi}^{p}(M, Y) \ni u \mapsto u-\xi \in L_{o}^{p}(M, \mathbb{B}),
\end{gathered}
$$

both of which are isometric.

Remark 3.17. If $Y_{i}$ and $Y$ are real Banach spaces (not necessarily isometric to each other) and if $\xi_{i}$ and $\xi$ are their origins, then the embeddings (3.4) are linear maps.

Definition 3.18 ( $L^{p}$-topology). We define the $L^{p}$-topology on

$$
\left(\bigsqcup_{i} L_{\xi_{i}}^{p}\left(M_{i}, Y_{i}\right)\right) \sqcup L_{\xi}^{p}(M, Y)
$$

as the restriction of that of $\left(\bigsqcup_{i} L_{o}^{p}\left(M_{i}, \mathbb{B}\right)\right) \sqcup L_{o}^{p}(M, \mathbb{B})$.

In the following, let us see that the $L^{p}$-topology defined here has some natural properties.

We need a lemma. $|A|$ denotes the measure of a measurable set $A$.

Lemma 3.19. (1) For any open subset $O \subset M$ we have

$$
\frac{\lim }{i}\left|\varphi_{i}^{-1}(O)\right| \geq|O| \text {. }
$$

(2) For any closed subset $F \subset M$ we have

$$
\varlimsup_{i}\left|\varphi_{i}^{-1}(F)\right| \leq|F| .
$$

We omit the proof of Lemma 3.19.

We give two measure approximations $\left\{\varphi_{i}: M_{i} \supset \mathcal{D}\left(\varphi_{i}\right) \rightarrow M\right\}$ and $\left\{\psi_{i}: M_{i} \supset\right.$ $\left.\mathcal{D}\left(\psi_{i}\right) \rightarrow M\right\}$.

Definition 3.20 (Equivalence relation between measure approximations). We say that $\left\{\varphi_{i}\right\}$ and $\left\{\psi_{i}\right\}$ are equivalent if for any compact subset $C \subset M$ and for any $\epsilon>0$ there exists $i(C, \epsilon)$ such that for any $i \geq i(C, \epsilon)$ and any $x \in \mathcal{D}\left(\varphi_{i}\right) \cap \mathcal{D}\left(\psi_{i}\right) \cap$ $\left(\varphi_{i}^{-1}(C) \cup \psi_{i}^{-1}(C)\right)$ we have $d_{M}\left(\varphi_{i}(x), \psi_{i}(x)\right)<\epsilon$, where $d_{M}$ is a distance function on $M$ compatible with the topology of $M$.

Note that the equivalence relation defined here is independent of the distance function $d_{M}$, because it can be described only by the uniform structure on $M$.

Lemma 3.21. If $\left\{\varphi_{i}\right\}$ and $\left\{\psi_{i}\right\}$ are equivalent, then the two $L^{p}$-topologies induced from $\left\{\varphi_{i}\right\}$ and $\left\{\psi_{i}\right\}$ coincide.

Proof. Assume that $\left\{\varphi_{i}\right\}$ and $\left\{\psi_{i}\right\}$ are equivalent. Take any $u \in C_{o}(M, \mathbb{B})$ and fix it. We set $C:=\operatorname{supp} u$. By the uniform continuity of $u$, for any $\epsilon>0$ there is $i(C, \epsilon)$ such that for any $x \in \mathcal{D}\left(\varphi_{i}\right) \cap \mathcal{D}\left(\psi_{i}\right) \cap\left(\varphi_{i}^{-1}(C) \cup \psi_{i}^{-1}(C)\right), i \geq i(C, \epsilon)$, we have $\left\|u \circ \varphi_{i}(x)-u \circ \psi_{i}(x)\right\|<\epsilon$ and therefore,

$$
d_{L^{p}}\left(\Phi_{i}(u), \Psi_{i}(u)\right)^{p} \leq \epsilon^{p}\left|\varphi_{i}^{-1}(C) \cup \psi_{i}^{-1}(C)\right|,
$$

where $\Psi_{i}$ is defined for $\psi_{i}$ in the same manner as (3.2). By using Lemma 3.19(2), this implies that $\varlimsup_{i} d_{L^{p}}\left(\Phi_{i}(u), \Psi_{i}(u)\right) \leq 2 \epsilon^{p}|C|$. By the arbitrariness of $\epsilon$ we have $\lim _{i} d_{L^{p}}\left(\Phi_{i}(u), \Psi_{i}(u)\right)=0$. Since this holds for any $u \in C_{o}(M, \mathbb{B})$, the two measure approximations $\left\{\Phi_{i}\right\}$ and $\left\{\Psi_{i}\right\}$ induce the same asymptotic relation between 
$L_{o}^{p}\left(M_{i}, \mathbb{B}\right)$ and $L_{o}^{p}(M, \mathbb{B})$. The restriction in Definition 3.18 has the same topology. This completes the proof.

Lemma 3.22. The $L^{p}$-topology of Definition 3.18 is independent of the Banach space $\mathbb{B}$ and the embedding $Y_{i}, Y \hookrightarrow \mathbb{B}$.

Proof. Assume we have two embeddings $\alpha: Y_{i}, Y \hookrightarrow \mathbb{B}_{\alpha}$ and $\beta: Y_{i}, Y \hookrightarrow \mathbb{B}_{\beta}$ into two real Banach spaces. Let $Z$ be the gluing of $\mathbb{B}_{\alpha}$ and $\mathbb{B}_{\beta}$ along the isometric images $\alpha(Y)$ and $\beta(Y)$, and $d_{Z}$ the metric on $Z$ defined by

$$
d_{Z}(x, y):= \begin{cases}\|x-y\| & \text { if } x, y \in \mathbb{B}_{\alpha} \text { or if } x, y \in \mathbb{B}_{\beta}, \\ \inf _{z \in \mathcal{Y}}(\|x-z\|+\|z-y\|) & \text { otherwise, }\end{cases}
$$

for $x, y \in Z$. Then we embed $\left(Z, d_{Z}\right)$ into a real Banach space $\mathbb{B}$ isometrically, so that we have the embeddings $Y_{i}, Y \hookrightarrow \mathbb{B}_{\alpha}, \mathbb{B}_{\beta} \hookrightarrow \mathbb{B}$. Identifying $\mathbb{B}_{\alpha}$ and $\mathbb{B}_{\beta}$ with their isometric images in $\mathbb{B}$, we assume that $\alpha$ and $\beta$ each take their values in $\mathbb{B}$. Let $u_{i} \in L_{\xi_{i}}^{p}\left(M_{i}, Y_{i}\right)$ and set

$$
\begin{gathered}
\hat{u}_{i}^{\alpha}:=\alpha \circ u_{i}, \hat{u}_{i}^{\beta}:=\beta \circ u_{i} \in L_{\xi_{i}}^{p}\left(M_{i}, \mathbb{B}\right), \\
u_{i}^{\alpha}:=\alpha \circ u_{i}-\alpha \circ \xi_{i}, u_{i}^{\beta}:=\beta \circ u_{i}-\beta \circ \xi_{i} \in L_{o}^{p}\left(M_{i}, \mathbb{B}\right) .
\end{gathered}
$$

Take a map $u \in C_{o}(M, \mathbb{B})$ with $|\operatorname{supp} u|>0$, and set $\hat{u}:=u+\xi$. Assume that

$$
\varlimsup_{i} d_{L^{p}}\left(u_{i}^{\alpha}, \Phi_{i}(u)\right)<\epsilon
$$

for a number $\epsilon>0$. Let us estimate $d_{L^{p}}\left(u_{i}^{\beta}, \Phi_{i}(u)\right)$ by $\epsilon$. We have

$$
\begin{aligned}
d_{L^{p}}\left(u_{i}^{\beta}, \Phi_{i}(u)\right)^{p}= & \int_{\varphi_{i}^{-1}(\operatorname{supp} u)}\left\|u_{i}^{\beta}(x)-u \circ \varphi_{i}(x)\right\|^{p} d x \\
& +\int_{M_{i} \backslash \varphi_{i}^{-1}(\operatorname{supp} u)}\left\|u_{i}^{\beta}(x)\right\|^{p} d x .
\end{aligned}
$$

Since $\left\|u_{i}^{\beta}(x)\right\|=d_{Y_{i}}\left(u_{i}(x), \xi_{i}(x)\right)=\left\|u_{i}^{\alpha}(x)\right\|$, the $\beta$ of the second term of the right hand-side of the above can be replaced by $\alpha$ and is less than $\epsilon^{p}$ for $i$ large enough. We estimate the first term. Note that, by (3.3), the limsup of the first term is less than or equal to

$$
\varlimsup_{i} \int_{\varphi_{i}^{-1}(\operatorname{supp} u)}\left\|\hat{u}_{i}^{\beta}(x)-\hat{u} \circ \varphi_{i}(x)\right\|^{p} d x .
$$

Let $R$ be a number with $R>\sup _{z \in \hat{u}(\operatorname{supp} u)}\left\|\alpha\left(y_{0}\right)-z\right\|$. Note that $\alpha\left(y_{0}\right)=\beta\left(y_{0}\right)$. There is $i(\epsilon)$ such that $\|\alpha(y)-\beta(y)\|<\epsilon|\operatorname{supp} u|^{-1 / p}$ for any $i \geq i(\epsilon)$ and any $y \in B\left(y_{i}, 2 R\right)\left(\subset Y_{i}\right)$. In particular, if a point $x \in M_{i}$ for $i \geq i(\epsilon)$ satisfies $u_{i}(x) \in$ $B\left(y_{i}, 2 R\right)$, then

$$
\left\|\hat{u}_{i}^{\alpha}(x)-\hat{u}_{i}^{\beta}(x)\right\|<\epsilon|\operatorname{supp} u|^{-1 / p} .
$$

Denote by $S_{\epsilon, i}$ the set of all $x \in \varphi_{i}^{-1}(\operatorname{supp} u)$ such that $u_{i}(x) \notin B\left(y_{i}, 2 R\right)$. Then, (3.6) holds for any $x \in M_{i} \backslash S_{\epsilon, i}, i \geq i(\epsilon)$. Since $\underline{\lim }_{i} \inf _{x \in S_{\epsilon, i}}\left\|\hat{u}_{i}^{\alpha}(x)-\alpha\left(y_{0}\right)\right\| \geq 2 R$ and $\hat{u} \circ \varphi_{i}(x) \in \hat{u}(\operatorname{supp} u)$, the triangle inequality implies that

$$
\frac{\lim }{i} \inf _{x \in S_{\epsilon, i}}\left\|\hat{u}_{i}^{\alpha}(x)-\hat{u} \circ \varphi_{i}(x)\right\| \geq R
$$


and so

$$
R^{p} \varlimsup_{i}\left|S_{\epsilon, i}\right| \leq \varlimsup_{i} \int_{S_{\epsilon, i}}\left\|\hat{u}_{i}^{\alpha}(x)-\hat{u} \circ \varphi_{i}(x)\right\|^{p} d x \leq \varlimsup_{i} d_{L^{p}}\left(u_{i}^{\alpha}, \Phi_{i}(u)\right)^{p}<\epsilon^{p} .
$$

Since $\left\|\hat{u}_{i}^{\alpha}(x)-\alpha\left(y_{i}\right)\right\|=\left\|\hat{u}_{i}^{\beta}(x)-\beta\left(y_{i}\right)\right\|$, we have

$$
\varlimsup_{i}\left|\left\|\hat{u}_{i}^{\alpha}(x)-\hat{u} \circ \varphi_{i}(x)\right\|-\left\|\hat{u}_{i}^{\beta}(x)-\hat{u} \circ \varphi_{i}(x)\right\|\right| \leq 2 R .
$$

By (3.6), (3.7), and (3.8), we see that (3.5) is

$$
\begin{aligned}
\leq & \varlimsup_{i}\left\{\int_{\varphi_{i}^{-1}(\operatorname{supp} u) \backslash S_{\epsilon, i}}\left(\left\|\hat{u}_{i}^{\alpha}(x)-\hat{u} \circ \varphi_{i}(x)\right\|+\epsilon|\operatorname{supp} u|^{-1 / p}\right)^{p} d x\right. \\
& \left.+\int_{\varphi_{i}^{-1}(\operatorname{supp} u) \cap S_{\epsilon, i}}\left(\left\|\hat{u}_{i}^{\alpha}(x)-\hat{u} \circ \varphi_{i}(x)\right\|+2 R\right)^{p} d x\right\} \\
\leq & \varlimsup_{i} \int_{\varphi_{i}^{-1}(\operatorname{supp} u)} 2^{p-1}\left\|\hat{u}_{i}^{\alpha}(x)-\hat{u} \circ \varphi_{i}(x)\right\|^{p} d x+2^{p-1}\left(\epsilon^{p}+2^{p} \epsilon^{p}\right) \\
\leq & \left(2^{p}+2^{2 p-1}\right) \epsilon^{p} .
\end{aligned}
$$

Since $\epsilon$ is arbitrary, we obtain

$$
\varlimsup_{i} d_{L^{p}}\left(u_{i}^{\beta}, \Phi_{i}(u)\right) \leq C_{p} \varlimsup_{i} d_{L^{p}}\left(u_{i}^{\alpha}, \Phi_{i}(u)\right),
$$

where $C_{p}$ is a constant depending only on $p$.

Now, assume that $u_{i}^{\alpha} \in L_{o}^{p}\left(M_{i}, \mathbb{B}\right) L^{p}$-converges to a map $u \in L_{o}^{p}(M, \mathbb{B})$. There is a continuous approximation $\tilde{u}_{j} \in C_{o}(M, \mathbb{B})$ of $u, j=1,2, \ldots$, such that $\left|\operatorname{supp} \tilde{u}_{j}\right|>$ 0 and $d_{L^{p}}\left(\tilde{u}_{j}, u\right) \rightarrow 0$ as $j \rightarrow \infty$. Since $u_{i}^{\alpha} \rightarrow u$ in $L^{p}$, we have

$$
\lim _{j} \varlimsup_{i} d_{L^{p}}\left(u_{i}^{\beta}, \Phi_{i}\left(\tilde{u}_{j}\right)\right) \leq C_{p} \lim _{j} \varlimsup_{i} d_{L^{p}}\left(u_{i}^{\alpha}, \Phi_{i}\left(\tilde{u}_{j}\right)\right)=0,
$$

which implies that $u_{i}^{\beta} L^{p}$-converges to $u$. This completes the proof.

Remark 3.23. The condition (3.3) depends on the embedding $Y_{i}, Y \hookrightarrow \mathbb{B}$. Assume that (3.3) holds for some embedding $Y_{i}, Y \hookrightarrow \mathbb{B}$, and take another embedding $Y_{i}, Y \hookrightarrow \mathbb{B}^{\prime}$. Then, replacing each $\mathcal{D}\left(\varphi_{i}\right)$ with a suitable subset, we can keep (3.3) for $Y_{i}, Y \hookrightarrow \mathbb{B}^{\prime}$. Since the restriction of $\varphi_{i}$ is equivalent to the original one, and by Lemma 3.21 , these induce the same $L^{p}$-topology.

Fix an embedding $Y_{i}, Y \hookrightarrow \mathbb{B}$. Let us prove that $\left(\bigsqcup_{i} L_{\xi_{i}}^{p}\left(M_{i}, Y_{i}\right)\right) \sqcup L_{\xi}^{p}(M, Y)$ is closed in $\left(\bigsqcup_{i} L_{o}^{p}\left(M_{i}, \mathbb{B}\right)\right) \sqcup L_{o}^{p}(M, \mathbb{B})$.

Lemma 3.24. Let $u_{i} \in L_{\xi_{i}}^{p}\left(M_{i}, Y_{i}\right)$ be a net such that $v_{i}:=u_{i}-\xi_{i}$ converges to a map $v \in L_{o}^{p}(M, \mathbb{B})$ in the $L^{p}$-topology. Then, $u:=v+\xi$ belongs to $L_{\xi}^{p}(M, Y)$.

Proof. Suppose that $u=v+\xi$ does not belong to $L_{\xi}^{p}(M, Y)$. Then, $\mid\{x \in M \mid$ $u(x) \notin Y\} \mid>0$. There is a small number $\delta>0$ such that $U:=\{x \in M \mid$ $\left.d_{\mathbb{B}}(u(x), Y)>\delta\right\}$ has positive measure, where $d_{\mathbb{B}}$ is the distance function induced from the Banach norm $\|\cdot\|$ on $\mathbb{B}$. For the $v$ and any $\epsilon>0$, there exists a continuous map $\tilde{v}_{\epsilon} \in C_{o}(M, \mathbb{B})$ such that $d_{L^{p}}\left(v, \tilde{v}_{\epsilon}\right)<\epsilon$. Setting $\tilde{u}_{\epsilon}:=\tilde{v}_{\epsilon}+\xi$ we have

$$
\begin{aligned}
\epsilon^{p} & >\int_{d_{\mathbb{B}}(u(x), Y)>\delta, d_{\mathbb{B}}\left(\tilde{u}_{\epsilon}(x), Y\right) \leq \delta / 2}\left\|u(x)-\tilde{u}_{\epsilon}(x)\right\|^{p} d x \\
& \geq(\delta / 2)^{p}\left|\left\{x \in U \mid d_{\mathbb{B}}\left(\tilde{u}_{\epsilon}(x), Y\right) \leq \delta / 2\right\}\right|
\end{aligned}
$$


and therefore

$$
\varliminf_{\epsilon \rightarrow 0+}\left|\left\{x \in M \mid d_{\mathbb{B}}\left(\tilde{u}_{\epsilon}(x), Y\right)>\delta / 2\right\}\right|>0 .
$$

Since $\tilde{u}_{\epsilon}$ is continuous, applying Lemma 3.19(1) yields

$$
\varliminf_{\epsilon \rightarrow 0+} \frac{\lim _{i}}{i}\left|\left\{x \in \mathcal{D}\left(\varphi_{i}\right) \mid d_{\mathbb{B}}\left(\tilde{u}_{\epsilon} \circ \varphi_{i}(x), Y\right)>\delta / 2\right\}\right|>0,
$$

which implies

$$
\varliminf_{\epsilon \rightarrow 0+} \frac{\lim }{i} \int_{\mathcal{D}\left(\varphi_{i}\right)}\left\|u_{i}(x)-\tilde{u}_{\epsilon} \circ \varphi_{i}(x)\right\|^{p} d x>0 .
$$

This is a contradiction to $v_{i} \rightarrow v$ in the $L^{p}$-topology and (3.3).

Lemma 3.25. Let $\left(Z, d_{Z}\right)$ be a separable metric space. For any closed subset $F \subset Z$ there exists a Borel map $\psi: Z \rightarrow F$ such that $d_{Z}(\psi(z), z) \leq 2 d_{Z}(z, F)$ for any $z \in Z$.

Proof. It is necessary that $\psi(z)=z$ for $z \in F$. We shall define $\psi$ on $Z \backslash F$. Take a dense countable subset $\left\{z_{n}\right\}_{n \in \mathbb{N}} \subset Z \backslash F$ and set $r_{n}:=d_{Z}\left(z_{n}, F\right)$ and $B_{n}:=B\left(z_{n}, r_{n} / 2\right) .\left\{B_{n}\right\}_{n \in \mathbb{N}}$ is an open covering of $Z \backslash F$. Let $B_{n}^{\prime}:=B_{n} \backslash \bigcup_{k=1}^{n-1} B_{k}$. Then, $\left\{B_{n}^{\prime}\right\}_{n \in \mathbb{N}}$ is a covering of $Z \backslash F$ consisting of disjoint Borel sets. For each $n \in \mathbb{N}$ there is a point $a_{n} \in F$ such that $d_{Z}\left(a_{n}, z_{n}\right)<3 r_{n} / 2$. We set $\psi(z):=a_{n}$ for $z \in B_{n}^{\prime}$. This defines a Borel map $\psi: Z \rightarrow F$. The triangle inequality shows that $d_{Z}(\psi(z), z) \leq 2 d_{Z}(z, F)$ for any $z \in Z$.

The following lemma verifies (A2) of Definition 3.1.

Lemma 3.26. For any $u \in L_{\xi}^{p}(M, Y)$ there exists a net $u_{i} \in L_{\xi_{i}}^{p}\left(M_{i}, Y_{i}\right)$ converging to $u$ in the $L^{p}$-topology.

Proof. Let $u \in L_{\xi}^{p}(M, Y)$. By Lemma 3.12, for any $\epsilon>0$ there exists a map $\tilde{u}_{\epsilon} \epsilon$ $C(M, \mathbb{B})$ such that $\tilde{u}_{\epsilon}-\xi \in C_{o}(M, \mathbb{B})$ and $d_{L^{p}}\left(\tilde{u}_{\epsilon}, u\right)^{p}<\epsilon$. Set $C_{\epsilon}:=\operatorname{supp}\left(\tilde{u}_{\epsilon}-\xi\right)$. Note that $C_{\epsilon}$ and $\tilde{u}_{\epsilon}\left(C_{\epsilon}\right)$ are compact. Applying Lemma 3.25 we have a Borel map $\psi_{i}: \mathbb{B} \rightarrow Y_{i}$ such that $\|\psi(y)-y\| \leq 2 d_{\mathbb{B}}\left(y, Y_{i}\right)$ for any $y \in \mathbb{B}$. We define, for $x \in M_{i}$,

$$
u_{\epsilon, i}(x):= \begin{cases}\psi_{i} \circ \tilde{u}_{\epsilon} \circ \varphi_{i}(x) & \text { if } x \in \varphi_{i}^{-1}\left(C_{\epsilon}\right), \\ \xi_{i}(x) & \text { if } x \in M_{i} \backslash \varphi_{i}^{-1}\left(C_{\epsilon}\right) .\end{cases}
$$

It then follows that $u_{\epsilon, i}\left(M_{i}\right) \subset Y_{i}$ and

$$
\begin{aligned}
& \int_{\mathcal{D}\left(\varphi_{i}\right)}\left\|u_{\epsilon, i}(x)-\tilde{u}_{\epsilon} \circ \varphi_{i}(x)\right\|^{p} d x \\
& =\int_{\varphi_{i}^{-1}\left(C_{\epsilon}\right)}\left\|\psi_{i} \circ \tilde{u}_{\epsilon} \circ \varphi_{i}(x)-\tilde{u}_{\epsilon} \circ \varphi_{i}(x)\right\|^{p} d x \\
& +\int_{\mathcal{D}\left(\varphi_{i}\right) \backslash \varphi_{i}^{-1}\left(C_{\epsilon}\right)}\left\|\xi_{i}(x)-\xi \circ \varphi_{i}(x)\right\|^{p} d x .
\end{aligned}
$$

By (3.3), the second term of the right-hand side tends to zero as $i \rightarrow \infty$. The first term is

$$
\leq 2^{p} \int_{\varphi_{i}^{-1}\left(C_{\epsilon}\right)} d_{\mathbb{B}}\left(\tilde{u}_{\epsilon}(x) \circ \varphi_{i}(x), Y_{i}\right)^{p} d x .
$$

Since $\tilde{u}_{\epsilon}\left(C_{\epsilon}\right)$ is compact and $\left(Y_{i}, y_{i}\right) \rightarrow\left(Y, y_{0}\right)$, we have

$$
\lim _{i} \sup _{y \in \tilde{u}_{\epsilon}\left(C_{\epsilon}\right)}\left|d_{\mathbb{B}}\left(y, Y_{i}\right)-d_{\mathbb{B}}(y, Y)\right|=0 .
$$


Therefore, the limit of (3.9) is

$$
\begin{aligned}
& =\lim _{i} 2^{p} \int_{\varphi_{i}^{-1}\left(C_{\epsilon}\right)} d_{\mathbb{B}}\left(\tilde{u}_{\epsilon}(x) \circ \varphi_{i}(x), Y\right)^{p} d x \\
& =2^{p} \int_{C_{\epsilon}} d_{\mathbb{B}}\left(\tilde{u}_{\epsilon}(x), Y\right)^{p} d x \leq 2^{p} d_{L^{p}}\left(\tilde{u}_{\epsilon}, u\right)^{p}<2^{p} \epsilon .
\end{aligned}
$$

Thus, setting $\hat{\Phi}_{i} u:=\Phi_{i}(u-\xi)+\xi_{i}$, we obtain

$$
\lim _{i} d_{L^{p}}\left(u_{\epsilon, i}, \hat{\Phi}_{i} \tilde{u}_{\epsilon}\right)^{p}=\lim _{i} \int_{\mathcal{D}\left(\varphi_{i}\right)}\left\|u_{\epsilon, i}(x)-\tilde{u}_{\epsilon} \circ \varphi_{i}(x)\right\|^{p} d x<2^{p} \epsilon,
$$

so that there exists a net of positive numbers $\epsilon_{i} \rightarrow 0$ such that

$$
d_{L^{p}}\left(u_{\epsilon_{i}, i}, \hat{\Phi}_{i} \tilde{u}_{\epsilon_{i}}\right)^{p}<2^{p} \epsilon_{i}
$$

for any $i$. Since $\hat{\Phi}_{i} \tilde{u}_{\epsilon_{i}}$ converges to $u$ in the $L^{p}$-topology, so does $u_{i}:=u_{\epsilon_{i}, i}$. This completes the proof.

We summarize Lemmas 3.22, 3.24, 3.26, and Remarks 3.23, 3.17 into the following:

Theorem 3.27. The $L^{p}$-topology on $\left(\bigsqcup_{i} L_{\xi_{i}}^{p}\left(M_{i}, Y_{i}\right)\right) \sqcup L_{\xi}^{p}(M, Y)$ defined in Definition 3.18 is

(1) independent of a representative $\left\{\varphi_{i}\right\}$ of the equivalence class of the measure approximations (but may depend on an equivalence class),

(2) independent of the Banach space $\mathbb{B}$ and the embedding $Y_{i}, Y \hookrightarrow \mathbb{B}$,

(3) an asymptotic relation.

Moreover, $\left(\bigsqcup_{i} L_{\xi_{i}}^{p}\left(M_{i}, Y_{i}\right)\right) \sqcup L_{\xi}^{p}(M, Y)$ is closed in $\left(\bigsqcup_{i} L_{o}^{p}\left(M_{i}, \mathbb{B}\right)\right) \sqcup L_{o}^{p}(M, \mathbb{B})$. If $Y_{i}$ and $Y$ are real Banach spaces and if $\xi_{i}$ and $\xi$ are their origins, then the $L^{p}$-topology is linear as an asymptotic relation.

For a pointed Gromov-Hausdorff convergent net $M_{i} \rightarrow M$ of proper pointed measured metric spaces, we have a unique associated equivalence class (as measure approximations) of measured pointed Gromov-Hausdorff approximations, so that the $L^{p}$-topology is uniquely determined.

We have the $L^{p}$-topology on the disjoint union

$$
\bigsqcup_{M,\left(Y, y_{0}\right)} L_{y_{0}}^{p}(M, Y)
$$

where $M$ runs over all isomorphic classes of pointed proper measured metric spaces, and $\left(Y, y_{0}\right)$ runs over all isometric classes of pointed proper metric spaces. We can think the union above as the fiber space over the product space $\left\{\left(M,\left(Y, y_{0}\right)\right)\right\}$ of $\{M\}$ and $\left\{\left(Y, y_{0}\right)\right\}$ with fibers $L_{y_{0}}^{p}(M, Y)$.

\section{VARiational CONVERGENCE OVER MEtriC SPACES}

We first give some definitions for variational convergences, e.g. asymptotic compactness and $\Gamma$-convergence, in Section 4.1. In the second section, 4.2, we prove the asymptotic compactness under a bound of the Poincaré constants and a bound of local covering orders, which is one of the main results of this paper. In the third section, 4.3, we prove the equivalence between the compact convergence and the Gromov-Hausdorff convergence of the energy-sublevel sets. 
4.1. Basics. Let $X_{i}$ and $X$ be metric spaces and an asymptotic relation between them be given. We take functions $E_{i}: X_{i} \rightarrow[0,+\infty]$ and $E: X \rightarrow[0,+\infty]$. The following is the Rellich compactness property.

Definition 4.1 (Compactness). We say that $E$ is compact if for any bounded net $x_{j} \in X$ with $\varlimsup E\left(x_{j}\right)<+\infty$ there exists a convergent subnet of $\left\{x_{j}\right\}$.

The next lemma is obvious.

Lemma 4.2. The following are equivalent:

(1) $E$ is lower semi-continuous and compact.

(2) $\{x \in X \mid E(x) \leq a\}$ is proper for any $a \geq 0$.

The Rellich compactness is generalized into the following.

Definition 4.3 (Asymptotic compactness, [27]). The net $\left\{E_{i}\right\}$ of functions is said to be asymptotically compact if for any bounded net $x_{i} \in X_{i}$ with $\varlimsup E_{i}\left(x_{i}\right)<+\infty$ there exists a convergent subnet of $\left\{x_{i}\right\}$.

Definition 4.4 ( $\Gamma$-convergence). We say that $E_{i} \Gamma$-converges to $E$ if the following are satisfied:

(Г1) For any $x \in X$ there exists a net $x_{i} \in X_{i}$ such that $x_{i} \rightarrow x$ and $E_{i}\left(x_{i}\right) \rightarrow$ $E(x)$

(Г2) If $X_{i} \ni x_{i} \rightarrow x \in X$, then $E(x) \leq \underline{\lim }_{i} E_{i}\left(x_{i}\right)$.

Definition 4.5 (Compact convergence). We say that $E_{i}$ compactly converges to $E$ if $E_{i} \Gamma$-converges to $E$ and if $\left\{E_{i}\right\}$ is asymptotically compact.

Lemma 4.6. If $E_{i} \Gamma$-converges to $E$, then $E$ is lower semi-continuous.

Proof. Let a sequence $x_{j} \in X, j \in \mathbb{N}$, converge to a point $x \in X$. By ( $\left.\Gamma 1\right)$, for each $j$ there is a net $x_{j, i} \in X_{i}$ converging to $x_{j}$ such that $\lim _{i} E_{i}\left(x_{j, i}\right)=E\left(x_{j}\right)$. There is a sequence $i(j) \rightarrow+\infty$ such that $\left|E_{i(j)}\left(x_{j, i(j)}\right)-E\left(x_{j}\right)\right|<1 / j$ and $x_{j, i(j)} \rightarrow x$. We have

$$
\frac{\varliminf_{j}}{} E\left(x_{j}\right)=\varliminf_{j} E_{i(j)}\left(x_{j, i(j)}\right) \geq E(x) .
$$

This completes the proof.

We have the following theorem in the same way as in the linear version, Lemma 2.14 of [22]. The proof is omitted.

Theorem 4.7. Assume that $X_{i}$ and $X$ are all separable. Then, for any $\left\{E_{i}\right\}$ there always exists a $\Gamma$-convergent subnet of $\left\{E_{i}\right\}$.

As an immediate consequence of the theorem, we have:

Corollary 4.8. Assume that $X_{i}$ and $X$ are all separable. If $\left\{E_{i}\right\}$ is asymptotically compact, it has a compactly convergent subnet.

The following is a generalization of Remark 2.6 of [22].

Proposition 4.9. Assume that $X$ is separable. If $E_{i}$ compactly converges to $E$, then $E$ is compact. 
Proof. Let $x_{j} \in X$ be a bounded net with $\varlimsup_{j} E\left(x_{j}\right)<+\infty$. By $(\Gamma 1)$, for each $j$ there is a net $x_{j, i} \in X_{i}$ such that, as $i \rightarrow \infty, x_{j, i} \rightarrow x_{j}$ and $E_{i}\left(x_{j, i}\right) \rightarrow E\left(x_{j}\right)$. Take a dense countable subset $\left\{\xi_{n}\right\}_{n \in \mathbb{N}} \subset X$ and, for each $n$, a net $\xi_{n, i} \in X_{i}$ with $\xi_{n, i} \rightarrow \xi_{n}$ as $i \rightarrow \infty$. Let us fix a net $N_{j} \in \mathbb{N}$ tending to $\infty$. By (A3), for any $j$ there is $i(j)$ such that $i(j) \rightarrow \infty$ as $j \rightarrow \infty$,

$$
\left|d_{X_{i}}\left(x_{j, i}, \xi_{n, i}\right)-d_{X}\left(x_{j}, \xi_{n}\right)\right|<1 / N_{j}, \quad \text { and } \quad\left|E_{i}\left(x_{j, i}\right)-E\left(x_{j}\right)\right|<1 / N_{j}
$$

for any $i \geq i(j)$ and $n=1,2, \ldots, N_{j}$. Since

$$
\varlimsup_{j} E_{i(j)}\left(x_{j, i(j)}\right)=\varlimsup_{j} E\left(x_{j}\right)<+\infty,
$$

the asymptotic compactness of $\left\{E_{i}\right\}$ implies that, by replacing $\{j\}$ by a sub-directed set, $\left\{x_{j, i(j)}\right\}_{j}$ converges to some point $x \in X$. It follows from (4.1) that

$$
\lim _{j} d_{X}\left(x_{j}, \xi_{n}\right)=d_{X}\left(x, \xi_{n}\right)
$$

for any $n \in \mathbb{N}$. For any $\epsilon>0$ there is $n \in \mathbb{N}$ such that $d_{X}\left(x, \xi_{n}\right)<\epsilon$ and hence $d_{X}\left(x_{j}, \xi_{n}\right)<\epsilon$ if $j$ is large enough. Therefore, by the triangle inequality, $d_{X}\left(x_{j}, x\right)<2 \epsilon$ for all sufficiently large $j$. This completes the proof.

Definition 4.10 (Asymptotic minimizer). An asymptotic minimizer of $\left\{E_{i}\right\}$ is defined to be a net $x_{i} \in X_{i}$ such that $\lim _{i}\left(E_{i}\left(x_{i}\right)-\inf E_{i}\right)=0$.

An asymptotic minimizer of $\left\{E_{i}\right\}$ always exists whenever $E_{i} \not \equiv+\infty$.

Proposition 4.11. Assume that $E \not \equiv+\infty$.

(1) If $E_{i} \Gamma$-converges to $E$ and if an asymptotic minimizer of $\left\{E_{i}\right\}$ converges to a point $x \in X$, then $x$ is a minimizer of $E$.

(2) If $E_{i}$ compactly converges to $E$, then any bounded asymptotic minimizer of $\left\{E_{i}\right\}$ has a subnet converging to a minimizer of $E$.

Proof. We omit the proof of (1) because it is easy.

Let us prove (2). Since there is a point $y \in X$ with $E(y)<+\infty,(\Gamma 1)$ implies that $\inf E_{i}$ is bounded. Let $x_{i} \in X_{i}$ be a bounded asymptotic minimizer of $\left\{E_{i}\right\}$. Then $E_{i}\left(x_{i}\right)$ is bounded. By the asymptotic compactness of $\left\{E_{i}\right\},\left\{x_{i}\right\}$ has a convergent subnet. By (1), its limit is a minimizer of $E$. This completes the proof.

4.2. Asymptotic compactness under a bound of Poincaré constants and local covering order. Let $M_{i}$ and $M$ be compact measured metric spaces such that $M_{i}$ converges to $M$ with respect to the measured Gromov-Hausdorff topology. Let $\left(Y_{i}, y_{i}\right)$ and $\left(Y, y_{0}\right)$ be pointed proper metric spaces such that $\left(Y_{i}, y_{i}\right)$ converges to $\left(Y, y_{0}\right)$ in the pointed Gromov-Hausdorff topology. By Definition 3.18, we have the $L^{p}$-topology on $\left(\bigsqcup_{i} L_{y_{i}}^{p}\left(M_{i}, Y_{i}\right)\right) \sqcup L_{y_{0}}^{p}(M, Y)$ for $p \geq 1$.

Remark 4.12. Since $M_{i}$ and $M$ are compact, for any maps $\xi_{i}: M_{i} \rightarrow Y_{i}$ and $\xi: M \rightarrow$ $Y$ as in Section 4.2 , by the compactness of $\xi(M)$ we have $L_{\xi}^{p}(M, Y)=L_{y_{0}}^{p}(M, Y)$ and there is $i_{0}$ such that $L_{\xi_{i}}^{p}\left(M_{i}, Y_{i}\right)=L_{y_{i}}^{p}\left(M_{i}, Y_{i}\right)$ for any $i \geq i_{0}$. The $L^{p}$-topologies on $\left(\bigsqcup_{i \geq i_{0}} L_{\xi_{i}}^{p}\left(M_{i}, Y_{i}\right)\right) \sqcup L_{\xi}^{p}(M, Y)$ and $\left(\bigsqcup_{i \geq i_{0}} L_{y_{i}}^{p}\left(M_{i}, Y_{i}\right)\right) \sqcup L_{y_{0}}^{p}(M, Y)$ coincide.

Let $E: L_{y_{0}}^{p}(M, Y) \rightarrow[0,+\infty]$ be a functional and set

$$
\mathcal{D}(E):=\left\{u \in L_{y_{0}}^{p}(M, Y) \mid E(u)<+\infty\right\} .
$$

Let $c, C, \rho$, and $R$ be constants with $c \geq 1, C>0$, and $0 \leq \rho \leq R$. $\mathcal{B}(M)$ denotes the family of Borel subsets of $M$. Consider the following condition, $(P)_{p, c, C, \rho, R}$. 
$(P)_{p, c, C, \rho, R}$ : For any $u \in \mathcal{D}(E)$, there exists a finitely subadditive function $\mu_{u}: \mathcal{B}(M) \rightarrow[0,+\infty)$ with $\mu_{u}(M) \leq E(u)$ such that

$$
\frac{1}{|B(x, r)|} \iint_{B(x, r) \times B(x, r)} d_{Y}(u(y), u(z))^{p} d y d z \leq C r^{p} \mu_{u}(B(x, c r))
$$

for any $x \in M$ and $r \in(\rho, R]$, where $|A|$ denotes the measure of $A \subset M$.

The inequality in $(P)_{p, c, C, \rho, R}$ is called a (weak) Poincaré inequality. Koskela, Shanmugalingam, and Tyson proved in [21] that $(P)_{2, c, C, 0, R}$ for $Y=\mathbb{R}$ implies $(P)_{2, c, C^{\prime}, 0, R}$ for general $Y$ under some natural assumption for $E$.

Definition 4.13 (Local covering order). Let $(S, d)$ be a locally compact metric space. Recall that the order of a covering $\mathcal{U}$ of $S$ at a point $x \in S$, say $\operatorname{ord}_{x} \mathcal{U}$, is the number of sets in $\mathcal{U}$ containing $x$ and that the order of $\mathcal{U}$ is defined to be $\sup _{x \in S} \operatorname{ord}_{x} \mathcal{U}$. For $c \geq 1$ and $r>0$, we denote by $K_{c, S}(r)$ the maximum of the order of the covering $\left\{B\left(x_{\lambda}, c r\right)\right\}_{\lambda}$, where $\left\{x_{\lambda}\right\}$ runs over all discrete subsets of $S$ such that $d\left(x_{\lambda}, x_{\lambda^{\prime}}\right) \geq r$ for all $\lambda \neq \lambda^{\prime}$. For a function $f(r)$, we say that the $c$-local covering order of $S$ is at most $f(r)$ if $K_{c, S}(r) \leq f(r)$ for any $r>0$.

The function $f(r)$ is usually taken as the Landau symbols $o\left(r^{q}\right)$ and $O\left(r^{q}\right)$, where $\lim _{r \rightarrow 0} o(r) / r=0$ and $\varlimsup_{r \rightarrow 0}|O(r)| / r<+\infty$.

The proof of the following lemma is straightforward and omitted.

Lemma 4.14. If a net of compact metric spaces $S_{i}$ Gromov-Hausdorff converges to a compact metric space $S$, then for any $r>0$,

$$
\varlimsup_{i} K_{c, S_{i}}(r) \leq K_{c, S}(r) .
$$

One of the main results of this paper is the following:

Theorem 4.15. Let $p, c, C$, and $R$ be positive constants with $p, c \geq 1$. Assume that the c-local covering order of $M$ is at most o $\left(r^{-p}\right)$ as $r \rightarrow 0$. Let $E_{i}: L_{y_{i}}^{p}\left(M_{i}, Y_{i}\right) \rightarrow$ $[0,+\infty]$ be a net of functionals such that each $E_{i}$ satisfies $(P)_{p, c, C, \rho_{i}, R}$ for some net $\rho_{i} \rightarrow 0$ with $0 \leq \rho_{i} \leq R$. Then, $\left\{E_{i}\right\}$ is asymptotically compact.

In Section 6, we give some interesting applications to the theorem.

As an immediate consequence of the theorem, we have the following Rellich compactness result.

Corollary 4.16. Let $p, c, C$, and $R$ be positive constants with $p, c \geq 1$. Assume that the c-local covering order of $M$ is at most $o\left(r^{-p}\right)$ as $r \rightarrow 0$. Let $E: L_{y_{0}}^{p}(M, Y) \rightarrow$ $[0,+\infty]$ be a functional satisfying $(P)_{p, c, C, 0, R}$. Then $E$ is compact.

Note that the doubling condition implies the boundedness of the $c$-local covering order, so that our assumption is weaker than the doubling condition. Under the doubling condition for the measure on $M$, Corollary 4.16 is well-known. According to a result by Cheeger [4], the Poincaré inequality and the doubling condition together imply that $M$ is essentially of finite dimension. Theorem 4.15 and Corollary 4.16 hold even for infinite-dimensional spaces, such as, the space $Q_{\infty}$ of the following example.

Example 4.17. Let us consider the infinite product $Q_{\infty}:=[0,1] \times[0,1 / 2] \times \cdots \times$ $\left[0,1 / 2^{n}\right] \times \cdots$ with $\ell^{2}$ norm. This is a compact metric space of infinite dimension. We observe that the $c$-local covering order of $Q_{\infty}$ is at most $O\left(r^{-\log _{2}(c+1)}\right)$. In fact, 
on $\mathbb{R}^{n}$ we have $K_{c, \mathbb{R}^{n}}(r) \leq(c+1)^{n}$ by calculating the volumes of balls. Considering $Q_{n}:=[0,1] \times[0,1 / 2] \times \cdots \times\left[0,1 / 2^{n}\right] \subset \mathbb{R}^{n}$ we have $K_{c, Q_{n}}(r) \leq K_{c, \mathbb{R}^{n}}(r) \leq$ $(c+1)^{n}$. There exists a universal constant $a>0$ such that $K_{c, Q_{\infty}}(r) \leq a K_{c, Q_{n}}(r)$ if $n \geq \log _{2}(1 / r)+a$. Thus we have $K_{c, Q_{\infty}}(r) \leq O\left(r^{-\log _{2}(c+1)}\right)$ by taking $n:=$ $\left[\log _{2}(1 / r)+a+1\right]$. Note that if $2^{p}>c+1$, then the assumption for the $c$-local covering order of Theorem 4.15 is satisfied for $M=Q_{\infty}$.

Let $\mu_{n}$ be the Lebesgue measure on $Q_{n}$ normalized as $\mu_{n}\left(Q_{n}\right)=1$ and $\mu_{\infty}$ the product probability measure on $Q_{\infty}$. Then, $Q_{n}$ measured Gromov-Hausdorff converges to $Q_{\infty}$. Under the Neumann condition, we define natural $p$-energy functionals $\mathcal{E}_{n}^{(p)}$ and $\mathcal{E}_{\infty}^{(p)}$ on $L^{p}\left(Q_{n}\right)$ and $L^{p}\left(Q_{\infty}\right)$ respectively as follows. For a smooth function $u: Q_{n} \rightarrow \mathbb{R}$,

$$
\mathcal{E}_{n}^{(p)}(u):=\int_{Q_{n}}\left(\sum_{k=1}^{n}\left(\frac{\partial u}{\partial x_{k}}\right)^{2}\right)^{\frac{p}{2}} d \mu_{n} .
$$

For a smooth cylindrical function $u: Q_{\infty} \rightarrow \mathbb{R}$ (i.e., $u$ is a function of the $Q_{n}$-factor for some $n \in \mathbb{N}$ ),

$$
\mathcal{E}_{\infty}^{(p)}(u):=\int_{Q_{\infty}}\left(\sum_{k=1}^{\infty}\left(\frac{\partial u}{\partial x_{k}}\right)^{2}\right)^{\frac{p}{2}} d \mu_{\infty},
$$

where the sum is in fact a finite sum. These are both closable (see [24]), and we denote their closure by the same notations $\mathcal{E}_{n}^{(p)}$ and $\mathcal{E}_{\infty}^{(p)}$. Then, $\mathcal{E}_{n}^{(p)} \Gamma$-converges to $\mathcal{E}_{\infty}^{(p)}$. We assume $p=2$. Then, the generator of $\mathcal{E}_{n}^{(2)}$ is the Laplacian on $Q_{n}$. The 1-local covering order of $M$ is at most $o\left(r^{-2}\right)$. We have $(P)_{2,1, C, 0, R}$ for $\mathcal{E}_{n}^{(2)}$ for some positive constants $C$ and $R$, where $C$ is independent of the dimension $n$ (see [1]). Thus, Theorem 4.15 implies that $\mathcal{E}_{n}^{(2)}$ compactly converges to $\mathcal{E}_{\infty}^{(2)}$. This compact convergence is also obtained in the following direct way. The eigenfunctions of the Laplacian on $Q_{n}$ form the products of one-dimensional eigenfunctions with Neumann boundary condition. They produce all the eigenfunctions of the generator of $\mathcal{E}_{\infty}^{(2)}$. By this, we can prove that $\mathcal{E}_{n}^{(2)}$ compactly converges to $\mathcal{E}_{\infty}^{(2)}$. The compact convergence also holds for an infinite Riemannian product $N_{1} \times N_{2} \times \cdots \times N_{n} \times \cdots$ of closed Riemannian manifolds $N_{n}$ such that the first nonzero eigenvalue of the Laplacian on $N_{n}$ is divergent to infinity as $n \rightarrow \infty$. Since the proof is elementary, we omit the details.

Remark 4.18. To Theorems 5.1, 5.2, and Corollary 5.1 of [22], we have to add the assumption that the $c$-local covering order of $X$ is at most $o\left(r^{-2}\right)$, which was missed there.

The basic strategy of the proof of Theorem 4.15 is the same as in the linear case in our previous paper [22]. However, we need much more delicate discussions and also recover a mistake in [22].

In [20], Korevaar and Schoen proved a Rellich-type compactness theorem; the proof does not seem to work to obtain our Theorem 4.15.

Proof of Theorem 4.15. We suppose the assumption of the theorem. As in Section 3.2 , we embed $\left(Y_{i}, y_{i}\right)$ and $\left(Y, y_{0}\right)$ into a Banach space $(\mathbb{B},\|\cdot\|)$ and consider the embeddings (3.4). We assume that $y_{0}=o$, the origin of $\mathbb{B}$. Let $u_{i} \in L_{y_{i}}^{p}\left(M_{i}, Y_{i}\right)$ be such that

$$
\sup _{i}\left(E_{i}\left(u_{i}\right)+d_{L^{p}}\left(u_{i}, y_{i}\right)^{p}\right)<\infty .
$$


Since $y_{i} \rightarrow y_{0}=o$ and $\left|M_{i}\right| \rightarrow|M|<\infty$, we have $\left\|\iota_{i}\left(u_{i}\right)-u_{i}\right\|_{L^{p}}=\left\|y_{i}\right\|\left|M_{i}\right|^{1 / p} \rightarrow$ 0 , where $\iota_{i}$ is the inclusion map in (3.4). Hence, $\left\|u_{i}\right\|_{L^{p}}$ is uniformly bounded. It suffices to prove that $\left\{u_{i}\right\}$ has an $L^{p}$-convergent subnet in the sense of Definition 3.14. Take a sequence of numbers $r_{j} \searrow 0, j=1,2, \ldots$, with $r_{j} \leq R$. For any $i$ and $j$, we find a maximal $r_{j}$-discrete net $\left\{x_{j k}^{i}\right\}_{k=1}^{N_{j}^{i}}$ of $M_{i}$, i.e., a maximal subset $\left\{x_{j k}^{i}\right\}_{k=1}^{N_{j}^{i}}$ of $M_{i}$ such that $d_{M_{i}}\left(x_{j k}^{i}, x_{j k^{\prime}}^{i}\right) \geq r_{j}$ for all $k \neq k^{\prime}$. Take a sequence of positive numbers $D_{j}$ such that

$$
D_{j}^{p}>\sup _{i, k} \frac{2\left\|u_{i}\right\|_{L^{p}}^{p}}{\left|B\left(x_{j k}^{i}, r_{j}\right)\right|}
$$

Note that since the measures on $M_{i}$ and $M$ are of full support, $\left|B\left(x_{j k}^{i}, r_{j}\right)\right|$ is bounded away from zero for all $i$ and $k$ if we fix $j$. Set

$$
B_{j k}^{i}:=\left\{x \in B\left(x_{j k}^{i}, r_{j}\right) \mid\left\|u_{i}(x)\right\|<D_{j}\right\}
$$

and define $\bar{u}_{j k}^{i} \in \mathbb{B}$ as the vector-valued integral

$$
\bar{u}_{j k}^{i}:=\frac{1}{\left|B_{j k}^{i}\right|} \int_{B_{j k}^{i}} u_{i}(x) d x .
$$

Claim 4.19. For any $i, j$, and $k$ with $\rho_{i}<r_{j}$, we have

$$
\int_{B\left(x_{j k}^{i}, r_{j}\right)}\left\|u_{i}(x)-\bar{u}_{j k}^{i}\right\|^{p} d x \leq 2 C r_{j}^{p} \mu_{u_{i}}\left(B\left(x_{j k}^{i}, c r_{j}\right)\right) .
$$

Proof. It follows that

$$
\left\|u_{i}\right\|_{L^{p}}^{p} \geq \int_{B_{j k}^{i}}\left\|u_{i}(x)\right\|^{p} d x \geq D_{j}^{p}\left|B_{j k}^{i}\right|
$$

which together with (4.2) implies $\left|B_{j k}^{i}\right|>\left|B\left(x_{j k}^{i}, r_{j}\right)\right| / 2$. Therefore, by Hölder's inequality and $(\mathrm{P})$, we have

$$
\begin{aligned}
& \int_{B\left(x_{j k}^{i}, r_{j}\right)}\left\|u_{i}(x)-\bar{u}_{j k}^{i}\right\|^{p} d x \\
& \leq \frac{1}{\left|B_{j k}^{i}\right|} \iint_{B\left(x_{j k}^{i}, r_{j}\right) \times B_{j k}^{i}}\left\|u_{i}(x)-u_{i}(y)\right\|^{p} d x d y \\
& \leq \frac{2}{\left|B\left(x_{j k}^{i}, r_{j}\right)\right|} \iint_{B\left(x_{j k}^{i}, r_{j}\right) \times B\left(x_{j k}^{i}, r_{j}\right)}\left\|u_{i}(x)-u_{i}(y)\right\|^{p} d x d y \\
& \leq 2 C r_{j}^{p} \mu_{u_{i}}\left(B\left(x_{j k}^{i}, c r_{j}\right)\right) .
\end{aligned}
$$

Set $U_{j k}^{i}:=B\left(x_{j k}^{i}, r_{j}\right) \backslash \bigcup_{l=1}^{k-1} B\left(x_{j l}^{i}, r_{j}\right)$. Note that $U_{j k}^{i} \cap U_{j l}^{i}=\emptyset$ for any $k \neq l$ and that $\bigcup_{k=1}^{N_{j}^{i}} U_{j k}^{i}=M_{i}$. We define a step map $\bar{u}_{j}^{i}: M_{i} \rightarrow \mathbb{B}$ by $\bar{u}_{j}^{i}:=\bar{u}_{j k}^{i}$ on each $U_{j k}^{i}$.

Claim 4.20. We have

$$
\lim _{j} \varlimsup_{i} d_{L^{p}}\left(u_{i}, \bar{u}_{j}^{i}\right)=0
$$


Proof. Claim 4.19 shows that if $\rho_{i}<r_{j}$, then

$$
\begin{aligned}
d_{L^{p}}\left(u_{i}, \bar{u}_{j}^{i}\right)^{p} & =\sum_{k=1}^{N_{j}^{i}} \int_{U_{j k}^{i}}\left\|u_{i}(x)-\bar{u}_{j k}^{i}\right\|^{p} d x \leq C r_{j}^{p} \sum_{k=1}^{N_{j}^{i}} \mu_{u_{i}}\left(B\left(x_{j k}^{i}, c r_{j}\right)\right) \\
& \leq C r_{j}^{p} K_{c, M_{i}}\left(r_{j}\right) \mu_{u_{i}}\left(M_{i}\right) .
\end{aligned}
$$

By Lemma 4.14 and the assumption, we have

$$
\varlimsup_{i} K_{c, M_{i}}\left(r_{j}\right) \leq K_{c, M}\left(r_{j}\right) \leq o\left(r_{j}^{-p}\right) .
$$

Since $\mu_{u_{i}}\left(M_{i}\right) \leq E_{i}\left(u_{i}\right)$ are bounded above, this completes the proof of Claim 4.20 .

For convenience in the later discussions, we define on a metric space $(S, d)$ a function $\rho[x, a, b]: S \rightarrow[0,1]$ for $a<b$ and $x \in S$ by

$$
\rho[x, a, b](y):= \begin{cases}1 & \text { if } d(x, y) \leq a, \\ (b-d(x, y)) /(b-a) & \text { if } a<d(x, y)<b, \\ 0 & \text { if } d(x, y) \geq b,\end{cases}
$$

for any $y \in S$. We see that $\rho[x, a, b]$ is a Lipschitz function with Lipschitz constant $1 /(b-a)$.

Claim 4.21. For any fixed $j$ and $k,\left\{\bar{u}_{j k}^{i}\right\}_{i}$ has a convergence subnet in $\mathbb{B}$.

Proof. We fix $j$ and $k$. Let $\epsilon$ be any number with $0<\epsilon<1$. Since $u_{i}(x) \epsilon$ $Y_{i} \cap B\left(o, D_{j}\right)$ for any $x \in B_{j k}^{i}$, there is a finite sequence of points $p_{1}^{i}, p_{2}^{i}, \ldots, p_{n(i)}^{i} \in$ $Y_{i} \cap B\left(o, D_{j}\right)$ depending on $\epsilon$ such that

$$
\left\|\bar{u}_{j k}^{i}-\frac{1}{n(i)} \sum_{l=1}^{n(i)} p_{l}^{i}\right\|<\epsilon .
$$

There is $i(\epsilon)$ such that $Y_{i} \cap B\left(o, D_{j}\right) \subset B(Y, \epsilon)$ for any $i \geq i(\epsilon)$. Let $i \geq i(\epsilon)$. We find $q_{l}^{i} \in Y$ such that $\left\|p_{l}^{i}-q_{l}^{i}\right\|<\epsilon$ and $\left\|q_{l}^{i}\right\|<D_{j}+\epsilon$ for any $l$. By the triangle inequalities, we have

$$
\left\|\bar{u}_{j k}^{i}-\frac{1}{n(i)} \sum_{l=1}^{n(i)} q_{l}^{i}\right\|<2 \epsilon
$$

so that $\bar{u}_{j k}^{i}$ for any $i \geq i(\epsilon)$ is in the $2 \epsilon$-neighborhood of the closed convex hull, say $C$, of $Y \cap \bar{B}\left(o, D_{j}+1\right)$. Consequently we have $\lim _{i} d_{\mathbb{B}}\left(\bar{u}_{j k}^{i}, C\right)=0$. We apply Mazur's compactness theorem which says that the closed convex hull of any compact subset of a Banach space is compact (see [26]). Since $Y \cap \bar{B}\left(o, D_{j}+1\right)$ is compact, $C$ is compact. This proves Claim 4.21.

There is a measure approximation $\left\{\varphi_{i}: \mathcal{D}\left(\varphi_{i}\right)=M_{i} \rightarrow M\right\}$ such that each $\varphi_{i}$ is an $\epsilon_{i}$-approximation for some $\epsilon_{i} \rightarrow 0+$. There is a sub-directed set $\mathcal{I}_{j}$ of $\{i\}$ depending on $j$ such that for every $k=1, \ldots, N_{j}^{i}$, the limits $x_{j k}:=\lim _{i} \varphi_{i}\left(x_{j k}^{i}\right)$, $N_{j}:=\lim _{i} N_{j}^{i}$, and $\bar{u}_{j k}:=\lim _{i} \bar{u}_{j k}^{i}$ all exist. Replacing it with a sub-directed set of $\mathcal{I}_{j}$, we assume that $N_{j}=N_{j}^{i}$ for all $i \in \mathcal{I}_{j}$. We may also assume that $\mathcal{I}_{j+1} \subset \mathcal{I}_{j}$ for any $j$. Therefore, by a diagonal argument, we find a common cofinal subnet of 
all $\mathcal{I}_{j}$ and denote it by $\mathcal{I}$. Set $U_{j k}:=B\left(x_{j k}, r_{j}\right) \backslash \bigcup_{l=1}^{k-1} B\left(x_{j l}, r_{j}\right)$. For any $\epsilon>0$, $x \in M$, and any set $A$, we define

$$
\begin{aligned}
\chi_{B\left(x, r_{j}\right)}^{\epsilon} & :=\rho\left[x, r_{j}-2 \epsilon, r_{j}-\epsilon\right]: M \rightarrow[0,1], \\
\chi_{U_{j k}}^{\epsilon} & :=\chi_{B\left(x_{j k}, r_{j}\right)}^{\epsilon} \cdot \prod_{l=1}^{k-1}\left(1-\chi_{B\left(x_{j l}, r_{j}\right)}^{\epsilon}\right): M \rightarrow[0,1], \\
I_{A}(x) & := \begin{cases}1 & \text { if } x \in A, \\
0 & \text { if } x \notin A .\end{cases}
\end{aligned}
$$

Claim 4.22. We have

$$
\begin{gathered}
\lim _{\epsilon \rightarrow 0+}\left\|\chi_{U_{j k}}^{\epsilon}-I_{U_{j k}}\right\|_{L^{p}(M)}=0, \\
\lim _{\epsilon \rightarrow 0+} \lim _{i \in \mathcal{I}}\left\|\chi_{U_{j k}}^{\epsilon} \circ \varphi_{i}-I_{U_{k}^{i}}\right\|_{L^{p}\left(M_{i}\right)}=0
\end{gathered}
$$

for any $j=1,2, \ldots$ and $k=1, \ldots, N_{j}$.

Proof. Take any $j=1,2, \ldots$ and $k=1, \ldots, N_{j}$ and fix them. Let $\epsilon$ be any positive number. We set $A\left(x, r, r^{\prime}\right):=B\left(x, r^{\prime}\right) \backslash B(x, r)$. Since $\left\{I_{U_{j k}} \neq \chi_{U_{j k}}^{\epsilon}\right\} \subset$ $\bigcup_{l=1}^{N_{j}} A\left(x_{j l}, r_{j}-2 \epsilon, r_{j}-\epsilon\right)$, we have

$$
\left\|I_{U_{j k}}-\chi_{U_{j k}}^{\epsilon}\right\|_{L^{p}(M)}^{p} \leq \sum_{l=1}^{N_{j}}\left|A\left(x_{j l}, r_{j}-2 \epsilon, r_{j}-\epsilon\right)\right|,
$$

which implies (4.3).

Let $i \in \mathcal{I}$ satisfy $\operatorname{dis} \varphi_{i}<\epsilon / 2$. For any $y \in M_{i}$ and $l=1, \ldots, N_{j}$, we have

$$
\left|d_{M_{i}}\left(x_{j l}^{i}, y\right)-d_{M}\left(x_{j l}, \varphi_{i}(y)\right)\right|<\epsilon / 2
$$

and hence $\left\{I_{U_{j k}^{i}} \neq \chi_{U_{j k}}^{\epsilon} \circ \varphi_{i}\right\} \subset \bigcup_{l=1}^{N_{j}} A_{l \epsilon}^{i}$, where $A_{l \epsilon}^{i}:=A\left(x_{j l}^{i}, r_{j}-3 \epsilon, r_{j}-\epsilon / 2\right)$, so that

$$
\left\|I_{U_{j k}^{i}}-\chi_{U_{j k}}^{\epsilon} \circ \varphi_{i}\right\|_{L^{p}\left(M_{i}\right)}^{p} \leq \sum_{l=1}^{N_{j}}\left|A_{l \epsilon}^{i}\right|
$$

Setting

$$
\alpha_{l \epsilon}:=\rho\left[x_{j l}, r_{j}-\epsilon / 4, r_{j}-\epsilon / 8\right] \cdot\left(1-\rho\left[x_{j l}, r_{j}-5 \epsilon, r_{j}-4 \epsilon\right]\right): M \rightarrow[0,1],
$$

we have $I_{A_{l \epsilon}^{i}} \leq \alpha_{l \epsilon} \circ \varphi_{i}$ for large $i$ and so

$$
\varlimsup_{i \in \mathcal{I}}\left|A_{l \epsilon}^{i}\right| \leq \varlimsup_{i \in \mathcal{I}} \int_{M_{i}} \alpha_{l \epsilon} \circ \varphi_{i} d x=\int_{M} \alpha_{l \epsilon} d x,
$$

which tends to zero as $\epsilon \rightarrow 0$. This completes the proof of the claim.

For $\bar{u}_{j k}=\lim _{i} \bar{u}_{j k}^{i}$ we define two maps $\bar{u}_{j}, \tilde{u}_{j}^{\epsilon}: M \rightarrow \mathbb{B}$ by

$$
\bar{u}_{j}(x):=\sum_{k=1}^{N_{j}} I_{U_{j k}}(x) \bar{u}_{j k} \quad \text { and } \quad \tilde{u}_{j}^{\epsilon}(x):=\sum_{k=1}^{N_{j}} \chi_{U_{j k}}^{\epsilon}(x) \bar{u}_{j k}, \quad x \in M .
$$


Claim 4.22 proves:

Claim 4.23. For any $j=1,2, \ldots$ we have

$$
\lim _{\epsilon \rightarrow 0+} d_{L^{p}}\left(\tilde{u}_{j}^{\epsilon}, \bar{u}_{j}\right)=0 \quad \text { and } \quad \lim _{\epsilon \rightarrow 0+} \varlimsup_{i} d_{L^{p}}\left(\Phi_{i} \tilde{u}_{j}^{\epsilon}, \bar{u}_{j}^{i}\right)=0,
$$

where $\Phi_{i}$ is defined by (3.2). Consequently, $\bar{u}_{j}^{i} L^{p}$-converges to $\bar{u}_{j}$.

Claim 4.24. $\left\{\bar{u}_{j}\right\}$ is Cauchy in $L_{o}^{p}(M, \mathbb{B})$.

Proof. By Claim 4.23 and (A3), for any $j$ and $j^{\prime}$,

$$
d_{L^{p}}\left(\bar{u}_{j}, \bar{u}_{j^{\prime}}\right)=\lim _{i} d_{L^{p}}\left(\bar{u}_{j}^{i}, \bar{u}_{j^{\prime}}^{i}\right) \leq \varlimsup_{i}\left(d_{L^{p}}\left(\bar{u}_{j}^{i}, u_{i}\right)+d_{L^{p}}\left(u_{i}, \bar{u}_{j^{\prime}}^{i}\right)\right),
$$

which tends to zero as $j, j^{\prime} \rightarrow \infty$ because of Claim 4.20.

We set $u:=\lim _{j \rightarrow \infty} \bar{u}_{j} \in L_{o}^{p}(M, \mathbb{B})$. Since $\bar{u}_{j}^{i} L^{p}$-converges to $\bar{u}_{j}$, by Claim 4.20, and by (A4), we obtain that $u_{i} L^{p}$-converges to $u$. This completes the proof of Theorem 4.15 .

4.3. Gromov-Hausdorff convergence of energy-sublevel sets. We give an asymptotic relation between metric spaces $X_{i}$ and $X$. Assume in this section that $X_{i}$ and $X$ are all separable. Define, for $c \in \mathbb{R}$,

$$
X^{c}:=\{x \in X \mid E(x) \leq c\}, \quad X_{i}^{c}:=\left\{x \in X_{i} \mid E_{i}(x) \leq c\right\},
$$

the $c$-sublevel sets of $E$ and $E_{i}$. The main purpose of this section is to prove:

Theorem 4.25. Assume that $E$ and $E_{i}$ are all compact, i.e., $X^{c}$ and $X_{i}^{c}$ are all proper for any $c \in \mathbb{R}$. Then the following are equivalent:

(1) $E_{i}$ compactly converges to $E$.

(2) For any $c \in \mathbb{R}$ there exist a net $c_{i} \searrow c$ of numbers and a net $o_{i} \in X_{i}$ of points converging to a point $o \in X$ such that the pointed space $\left(X_{i}^{c_{i}}, o_{i}\right)$ converges to $\left(X^{c}, o\right)$ in the Gromov-Hausdorff topology which is compatible with the asymptotic relation between $\left\{X_{i}\right\}$ and $X$.

Condition (2) of the theorem corresponds to the spectral concentration due to Gromov (see Section $3 \frac{1}{2} .57$ of [8]).

We give a simple example.

Example 4.26. Let $M_{i}:=\left\{a_{i}, b_{i}\right\}$ be a net of metric spaces consisting of two different points $a_{i}$ and $b_{i}$ such that $d_{M_{i}}\left(a_{i}, b_{i}\right) \rightarrow 0$, and let $M:=\{a\}$ be a space consisting of a single point $a$. We equip $M_{i}$ and $M$ with measures $d x_{i}:=\alpha d \delta_{a_{i}}+$ $\beta d \delta_{b_{i}}$ and $d x:=(\alpha+\beta) d \delta_{a}$ respectively, where $\alpha$ and $\beta$ are positive constants and $\delta_{z}$ denotes Dirac's $\delta$-measure at a point $z$. $M_{i}$ converges to $M$ with respect to the measured Gromov-Hausdorff topology. For a pointed proper metric space $\left(Y, y_{0}\right)$, let us consider $X_{i}:=L_{y_{0}}^{2}\left(M_{i}, Y\right)$ and $X:=L_{y_{0}}^{2}(M, Y) . X$ is isometric to $\sqrt{\alpha+\beta} Y$, where $c Y$ means the space $Y$ with metric multiplied by a positive constant $c$. It follows that

$$
d_{L^{2}}(u, v)^{2}=\alpha d_{Y}\left(u\left(a_{i}\right), v\left(a_{i}\right)\right)^{2}+\beta d_{Y}\left(u\left(a_{i}\right), v\left(a_{i}\right)\right)^{2}, \quad u, v \in X_{i} .
$$

Therefore, $X_{i}$ is isometric to the product $\sqrt{\alpha} Y \times \sqrt{\beta} Y$. We have the map $\varphi_{i}$ : $M_{i} \rightarrow M$ defined by $\varphi\left(a_{i}\right):=\varphi\left(b_{i}\right):=a$, which induces a map $\Phi_{i}: X \rightarrow X_{i}$, $\Phi_{i}(u):=u \circ \varphi_{i}$. This coincides with the diagonal embedding

$$
\sqrt{\alpha+\beta} Y \hookrightarrow \sqrt{\alpha} Y \times \sqrt{\beta} Y, \quad y \mapsto(y, y) .
$$


We define natural energy functionals on $X_{i}$ and $X$ as follows:

$$
E_{i}(u):=\frac{d_{Y}\left(u\left(a_{i}\right), u\left(b_{i}\right)\right)^{2}}{d_{M_{i}}\left(a_{i}, b_{i}\right)^{2}}, \quad E(v):=0
$$

for any $u \in X_{i}$ and $v \in X$. Then, we observe that for a fixed number $c \geq 0, X_{i}^{c}$ shrinks to $X^{c}=X=\sqrt{\alpha+\beta} Y$.

For the proof of the theorem we present some definitions and notations.

Definition $4.27\left(\underline{\lim }_{i} A_{i}, \varlimsup_{i} A_{i}\right)$. Let $A_{i} \subset X_{i}$. We define two subsets $\underline{\lim }_{i} A_{i}$ and $\overline{\lim }_{i} A_{i}$ of $X$ by the following:

(LI) $x \in \underline{\lim }_{i} A_{i}$ iff there exists a net $x_{i} \in A_{i}$ converging to $x$.

(LS) $x \in \overline{\overline{\lim }}_{i} A_{i}$ iff there exist a sub-directed set $\{j\}$ of $\{i\}$ and a net $x_{j} \in A_{j}$ such that $x_{j} \rightarrow x$.

It is obvious that $\underline{\lim }_{i} A_{i} \subset \varlimsup_{i} A_{i}$.

Notation 4.28. Let $c \in \mathbb{R}$ be any number. We define

$$
\underline{X}^{c}:=\varliminf_{i} X_{i}^{c} \quad \text { and } \quad \bar{X}^{c}:=\varlimsup_{i} X_{i}^{c} .
$$

Let $o_{i} \in X_{i}$ be a net converging to a point $o \in X$. For $r>0$, we denote by $\bar{B}(o, r)$ the closure of the ball $B(o, r)$ and define

$$
\begin{array}{rlrl}
X_{i}^{c}(r) & :=X_{i}^{c} \cap \bar{B}\left(o_{i}, r\right), & X^{c}(r):=X^{c} \cap \bar{B}(o, r), \\
\underline{X}^{c}(r):=\underline{X}^{c} \cap \bar{B}(o, r), & \bar{X}^{c}(r):=\bar{X}^{c} \cap \bar{B}(o, r) .
\end{array}
$$

To prove the implication $(1) \Rightarrow(2)$ of Theorem 4.25 , we need some lemmas.

Lemma 4.29. Under (1) of Theorem 4.25, we have

$$
\bigcap_{c^{\prime}>c} \underline{X}^{c^{\prime}}=\bigcap_{c^{\prime}>c} \bar{X}^{c^{\prime}}=X^{c}
$$

for any $c \in \mathbb{R}$.

Proof. By using ( $\Gamma 1)$, it is easy to show that $\underline{X}^{c^{\prime}} \supset X^{c}$ for any $c<c^{\prime}$, and hence

$$
\bigcap_{c^{\prime}>c} \underline{X}^{c^{\prime}} \supset X^{c}
$$

Take any $x \in \bigcap_{c^{\prime}>c} \bar{X}^{c^{\prime}}$. For the lemma it suffices to show that $x \in X^{c}$. In fact, for any $c^{\prime}>c$, since $x \in \bar{X}^{c^{\prime}}$, there exist a sub-directed set $\{j\}$ of $\{i\}$ and a net $x_{j} \in X_{j}^{c^{\prime}}$ such that $x_{j} \rightarrow x$. By $(\Gamma 2)$ we have

$$
E(x) \leq \frac{\lim }{j} E_{j}\left(x_{j}\right) \leq c^{\prime} .
$$

By the arbitrariness of $c^{\prime}$ with $c^{\prime}>c$, we obtain $x \in X^{c}$. This completes the proof of the lemma.

Lemma 4.30. Under (1) of Theorem 4.25, for any $c \in \mathbb{R}$ and $r>0, \bar{X}^{c}(r)$ is compact.

Proof. By Lemma 4.29, $\bar{X}^{c}$ is contained in $X^{c}$. It follows from a diagonal argument that $\bar{X}^{c}$ is a closed subset of $X$. Since $X^{c}$ is proper, $\bar{X}^{c}(r)$ is compact. 
Lemma 4.31. Under (1) of Theorem 4.25, for any $c \in \mathbb{R}, \epsilon>0$ and $r>r_{0}>0$, there exists $c^{\prime}=c^{\prime}\left(c, \epsilon, r, r_{0}\right)>c$ such that

$$
X^{c}\left(r_{0}\right) \subset \underline{X}^{c^{\prime}}\left(r_{0}\right) \subset \bar{X}^{c^{\prime}}(r) \subset B\left(X^{c}(r), \epsilon\right) .
$$

Proof. Lemma 4.29 implies that $X^{c}\left(r_{0}\right) \subset \underline{X}^{c^{\prime}}\left(r_{0}\right)$ for any $c^{\prime}>c$, and $\underline{X}^{c^{\prime}}\left(r_{0}\right) \subset$ $\bar{X}^{c^{\prime}}(r)$ is obvious. Thus, it suffices to prove $\bar{X}^{c^{\prime}}(r) \subset B\left(X^{c}(r), \epsilon\right)$ for some $c^{\prime}$ with $c^{\prime}>c$. Suppose the contrary, so that there exists a sequence $c_{j} \searrow c$ of numbers and a sequence $x_{j} \in \bar{X}^{c_{j}}(r) \backslash B\left(X^{c}(r), \epsilon\right)$. By Lemma 4.30, $x_{j}$ has a convergent subsequence and we may assume that $x_{j}$ converges to a point $x \in X$. Then $x$ belongs to $\bigcap_{j=1}^{\infty} \bar{X}^{c_{j}}(r) \backslash B\left(X^{c}(r), \epsilon\right)$. However, by Lemma 4.29, $\bigcap_{j=1}^{\infty} \bar{X}^{c_{j}}(r)=X^{c}$. This is a contradiction and completes the proof.

Lemma 4.32. Assume (1) of Theorem 4.25 and let $\left\{f_{i}: X \rightarrow X_{i}\right\}$ be asymptotically continuous metric approximation compatible with the asymptotic relation between $\left\{X_{i}\right\}$ and $X$ (such a $\left\{f_{i}\right\}$ always exists by Lemma 3.7). Then for any $c^{\prime}>c$ and $\epsilon, r>0$ there exists $i_{0}=i_{0}\left(c, c^{\prime}, \epsilon, r\right)$ such that

$$
X_{i}^{c}(r) \subset B\left(f_{i}\left(\bar{X}^{c^{\prime}}(r)\right), \epsilon\right)
$$

for any $i \geq i_{0}$.

Proof. Suppose not. Then, there exists a net $x_{i} \in X_{i}^{c}(r)$ such that

$$
d_{X_{i}}\left(x_{i}, f_{i}\left(\bar{X}^{c^{\prime}}(r)\right)\right) \geq \epsilon .
$$

Since $\left\{E_{i}\right\}$ is asymptotically compact, $x_{i}$ has a convergent subnet and we denote its limit by $x$. It follows that $x$ belongs to $\bar{B}(o, r)$. By recalling the definition of $\bar{X}^{c}$, we have $x \in \bar{X}^{c}(r)$. Since $d_{X_{i}}\left(x_{i}, f_{i}(x)\right) \rightarrow 0$, this contradicts (4.5).

Lemma 4.33. Under (1) of Theorem 4.25, for any $c^{\prime}>c, \epsilon>0$, and $r>r_{0}>0$ there exists $i_{1}=i_{1}\left(c, c^{\prime}, \epsilon, r, r_{0}\right)$ such that

$$
f_{i}\left(\underline{X}^{c^{\prime}}\left(r_{0}\right)\right) \subset B\left(X_{i}^{c^{\prime}}(r), \epsilon\right)
$$

for any $i \geq i_{1}$.

Proof. Suppose not. Then there exists a point $x \in \underline{X}^{c^{\prime}}\left(r_{0}\right)$ such that

$$
d_{X_{i}}\left(f_{i}(x), X_{i}^{c^{\prime}}(r)\right) \geq \epsilon .
$$

By the definition of $\underline{X}^{c^{\prime}}$, there exists a net $x_{i} \in X_{i}^{c^{\prime}}$ converging to $x$. Since $d_{X}(o, x) \leq r_{0}<r$, if $i$ is sufficiently large, $x_{i}$ belongs to $X_{i}^{c^{\prime}}(r)$. We also have $d_{X_{i}}\left(f_{i}(x), x_{i}\right) \rightarrow 0$, which is a contradiction.

Proof of Theorem 4.25. Let us first prove $(1) \Rightarrow(2)$. We assume (1). Let $r_{0}>$ 0 . Since $\bigcap_{r>r_{0}} X^{c}(r)=X^{c}\left(r_{0}\right)$, for any $\epsilon>0$ there exists $r>r_{0}$ such that $d_{H}\left(X^{c}(r), X^{c}\left(r_{0}\right)\right)<\epsilon$. Combining this with Lemmas 4.31, 4.32, and 4.33 proves

$$
\varlimsup_{i} d_{G H}\left(X^{c}\left(r_{0}\right), X_{i}^{c^{\prime}}(r)\right)<4 \epsilon .
$$

Therefore, by taking a sequence $\epsilon_{j} \searrow 0$, there exist sequences $r_{j} \searrow r_{0}$ and $c_{j} \searrow c$ such that

$$
\varlimsup_{i} d_{G H}\left(X^{c}\left(r_{0}\right), X_{i}^{c_{j}}\left(r_{j}\right)\right)<\epsilon_{j}
$$


so that we can find a divergent sequence $i(j)$ such that for any $i \geq i(j)$,

$$
d_{G H}\left(X^{c}\left(r_{0}\right), X_{i}^{c_{j}}\left(r_{j}\right)\right)<\epsilon_{j} .
$$

Therefore, there exists a monotone increasing divergent net $\{j(i)\}$ such that

$$
d_{G H}\left(X^{c}\left(r_{0}\right), X_{i}^{c_{j(i)}}\left(r_{j(i)}\right)\right)<\epsilon_{j(i)},
$$

which implies (2).

Let us prove $(2) \Rightarrow(1)$. Assume (2). To prove the asymptotic compactness of $\left\{E_{i}\right\}$, we take a net $x_{i} \in X_{i}^{c}(r)$ for some fixed $c \in \mathbb{R}$ and $r>0$. It suffices to prove that $x_{i}$ has a convergent subnet. In fact, since $\left(X_{i}^{c_{i}}, o_{i}\right)$ converges to $\left(X^{c}, o\right)$ in the Gromov-Hausdorff topology for some $c_{i} \searrow c$ and since $x_{i}$ belongs to $X_{i}^{c_{i}}$, it has a convergent subnet.

To prove $(\Gamma 2)$, we take a net $x_{i} \in X_{i}$ converging to a point $x \in X$. We may assume that $\varlimsup_{i} E_{i}\left(x_{i}\right)<+\infty$. Let $c$ be any number with $c>\underline{\lim }_{i} E_{i}\left(x_{i}\right)$. There exists a subnet $\{j\}$ of $\{i\}$ such that $\underline{\lim }_{i} E_{i}\left(x_{i}\right)=\lim _{j} E_{j}\left(x_{j}\right)$ and $E_{j}\left(x_{j}\right)<c$ for any $j$. By the assumption, $\left(X_{j}^{c_{j}}, o_{i}\right)$ converges to $\left(X^{c}, o\right)$ for some net $c_{j} \searrow c$. Since $x_{j}$ belongs to $X_{j}^{c_{j}}, x$ belongs to $X^{c}$. By the arbitrariness of $c$, we have $E(x) \leq \underline{\lim }_{i} E_{i}\left(x_{i}\right)$.

Finally we shall prove $(\Gamma 1)$. Let $x \in X$. If $E(x)=+\infty$, then (A2) of Definition 3.1 tells us to find a net $x_{i} \in X_{i}$ converging to $x$ and we have $E_{i}\left(x_{i}\right) \rightarrow E(x)$ by $(\Gamma 2)$. Assume $E(x)<+\infty$ and set $c:=E(x)$. Since there exists a net $c_{i} \searrow c$ such that $\left(X_{i}^{c_{i}}, o_{i}\right)$ converges to $\left(X^{c}, o\right)$, we can find a net $x_{i} \in X_{i}^{c_{i}}$ converging to $x$. Then we have $E_{i}\left(x_{i}\right) \leq c_{i} \searrow c=E(x)$, which together with (Г2) shows $E\left(x_{i}\right) \rightarrow E(x)$. This completes the proof of Theorem 4.25.

Definition 4.34 (Essentially critical value). A value $c$ of $E$ is said to be essentially critical if the closure of the set $\{x \in X \mid E(x)<c\}$ is a proper subset of $X^{c}$.

A metric space is called a geodesic space if any two points in the space is joined by a length-minimizing curve, called a geodesic, and the distance between them coincides with the length of the geodesic.

Proposition 4.35. If $X$ is a geodesic space and if $E: X \rightarrow[0,+\infty]$ is a convex function, then any nonminimal value of $E$ is not essentially critical.

Proof. Let $c \in \mathbb{R}$ be a nonminimal value of $E$ and take a point $x \in X$ with $E(x)=c$. It suffices to prove that there exists a sequence in $\{E<c\}$ converging to $x$. In fact, since $c$ is not minimal, we can find a point $y \in X$ with $E(y)<c$ and join $x$ and $y$ by a geodesic segment $\gamma$. It follows from the convexity of $E$ that $\gamma \backslash\{x\}$ is contained in $\{E<c\}$. This completes the proof.

Theorem 4.36. Assume that $E$ and $E_{i}$ are all compact. If $E_{i}$ compactly converges to $E$ and if a number $c \in \mathbb{R}$ is not an essentially critical value of $E$, then for any net $c_{i} \rightarrow c$ (not necessarily monotone), $\left(X_{i}^{c_{i}}, o_{i}\right)$ converges to $\left(X^{c}, o\right)$ in the Gromov-Hausdorff topology compatible with the asymptotic relation between $\left\{X_{i}\right\}$ and $X$, where $o_{i} \in X_{i}$ is any net converging to a point $o \in X$.

Proof. Since $c$ is not essentially critical, we have

$$
X^{c}=\overline{\{E<c\}}=\overline{\bigcup_{\delta>0} X^{c-\delta}},
$$


and hence, as $\delta \searrow 0, X^{c-\delta}$ converges to $X^{c}$ in the compact Hausdorff convergence. On the other hand, since

$$
X^{c}=\bigcap_{\delta>0} X^{c+\delta}
$$

as $\delta \searrow 0, X^{c+\delta}$ converges to $X^{c}$ in the compact Hausdorff convergence. Applying Theorem 4.25 yields that for any $\delta>0$ there exist two nets $\rho_{i} \searrow 0$ and $\rho_{i}^{\prime} \searrow 0$ such that $\left(X_{i}^{c-\delta+\rho_{i}}, o_{i}\right) \rightarrow\left(X^{c-\delta}, o\right)$ and $\left(X_{i}^{c+\delta+\rho_{i}^{\prime}}, o_{i}\right) \rightarrow\left(X^{c+\delta}, o\right)$ in the GromovHausdorff topology, where $o_{i} \in X$ is a net converging to a point $o \in X$. Let $c_{i}$ be any net of numbers converging to $c$. For all sufficiently large $i$, we have $c-\delta+\rho_{i}<c_{i}<c+\delta+\rho_{i}^{\prime}$ and so $X_{i}^{c-\delta+\rho_{i}} \subset X_{i}^{c_{i}} \subset X_{i}^{c+\delta+\rho_{i}^{\prime}}$. Therefore, $\left(X_{i}^{c_{i}}, o_{i}\right)$ converges to $\left(X^{c}, o\right)$ in the Gromov-Hausdorff topology.

\section{VARiational CONVERGENCE OVER CAT(0)-SPACES}

5.1. CAT(0)-spaces. A geodesic in a metric space is a rectifiable curve joining two points $x$ and $y$ in the metric space that is length-minimizing among all curves joining $x$ and $y$. Assume that all geodesics have parameters proportional to arclength. A geodesic space is a metric space any two points of which can be joined by a geodesic and the distance between them coincides with the length of the geodesic. Note that a geodesic joining two fixed points is not necessarily unique. We denote by $x y$ one of geodesics joining $x$ to $y$. A triangle $\triangle x y z$ in a geodesic space consists of three edges, $x y, y z$, and $z x$. For any triangle $\triangle x y z$ there is a triangle $\triangle \tilde{x} \tilde{y} \tilde{z}$ in $\mathbb{R}^{2}$ having same side lengths as $\triangle x y z$. We call such a $\triangle \tilde{x} \tilde{y} \tilde{z}$ a comparison triangle of $\triangle x y z$. A geodesic space $\left(H, d_{H}\right)$ is called a CAT(0)-space if the following triangle comparison condition is satisfied:

(T) Take any triangle $\triangle x y z$ in $H$ and a comparison triangle $\triangle \tilde{x} \tilde{y} \tilde{z}$ of it. For any points $a \in x y, b \in x z, \tilde{a} \in \tilde{x} \tilde{y}$, and $\tilde{b} \in \tilde{x} \tilde{z}$ with $d_{H}(x, a)=d_{H}(\tilde{x}, \tilde{a})$ and $d_{H}(x, b)=d_{H}(\tilde{x}, \tilde{b})$, we have $d_{H}(a, b) \leq d_{H}(\tilde{a}, \tilde{b})$.

Let $H$ be a complete CAT( 0$)$-space. It follows from (T) that for two given points $x, y \in H$, a geodesic $x y$ is unique. However geodesics in $H$ may branch in general. Let $C \subset H$ be a closed convex set. Then for any $x \in H$ there exists a unique point in $C$ nearest to $x$, which we denote by $\pi_{C}(x)$. The map $\pi_{C}: H \rightarrow C$ is 1-Lipschitz (see II.2 of [2]).

If $M$ is a measure space and if $Y$ is $\operatorname{CAT}(0)$, then $L_{\xi}^{2}(M, Y)$ is $\operatorname{CAT}(0)$ for any measurable map $\xi: M \rightarrow Y$ (see [13]).

Assume that an asymptotic relation between metric spaces $\left\{X_{i}\right\}$ and $X$ is given. Consider the following condition:

(G) If $\gamma_{i}:[0,1] \rightarrow X_{i}$ and $\gamma:[0,1] \rightarrow X$ are geodesics such that $\gamma_{i}(0) \rightarrow \gamma(0)$ and $\gamma_{i}(1) \rightarrow \gamma(1)$, then $\gamma_{i}(t) \rightarrow \gamma(t)$ for any $t \in[0,1]$.

\section{Proposition 5.1.}

(1) If $(\mathrm{G})$ is satisfied and if each $X_{i}$ is geodesic (resp. CAT(0)), then so is $X$.

(2) If each $X_{i}$ is $\mathrm{CAT}(0)$ and $X$ is geodesic, then $(\mathrm{G})$ is satisfied and $X$ is $\operatorname{CAT}(0)$.

Proof. We omit (1) and prove only (2). Let $\gamma_{i}:[0,1] \rightarrow X_{i}$ and $\gamma:[0,1] \rightarrow X$ be geodesics such that $\gamma_{i}(0) \rightarrow \gamma(0)$ and $\gamma_{i}(1) \rightarrow \gamma(1)$. By (A2), for a given $t \in[0,1]$ there exists a net $x_{i} \in X_{i}$ converging to $\gamma(t)$. It follows from (A3) that $d_{X_{i}}\left(\gamma_{i}(0), x_{i}\right) \rightarrow t l$ and $d_{X_{i}}\left(x_{i}, \gamma_{i}(1)\right) \rightarrow(1-t) l$, where $l:=d_{X}(\gamma(0), \gamma(1))$. 
Applying the triangle comparison condition, (T), to $\triangle x_{i} \gamma_{i}(0) \gamma_{i}(1)$ shows that $d_{X_{i}}\left(x_{i}, \gamma_{i}(t)\right) \rightarrow 0$. Thus, by (A4), we have $\gamma_{i}(t) \rightarrow \gamma(t)$. We have obtained $(\mathrm{G})$. By using (1), $X$ is $\mathrm{CAT}(0)$.

5.2. Weak convergence. Let $H_{i}$ and $H$ be complete separable CAT(0)-spaces that have an asymptotic relation. We have $(\mathrm{G})$ by Proposition 5.1(2). In what follows, strong convergence means convergence with respect to the asymptotic relation.

Definition 5.2 (Weak convergence). We say that a net $x_{i} \in H_{i}$ weakly converges to a point $x \in H$ if for any net of geodesic segments $\gamma_{i}$ in $H_{i}$ strongly converging to a geodesic segment $\gamma$ in $H$ with $\gamma(0)=x, \pi_{\gamma_{i}}\left(x_{i}\right)$ strongly converges to $x$.

It is easy to prove that a strong convergence implies a weak convergence and that a weakly convergent net always has a unique weak limit.

Lemma 5.3. Assume that $H_{i} \ni x_{i} \rightarrow x \in H$ weakly and $H_{i} \ni y_{i} \rightarrow y \in H$ strongly. Then, we have

(1) $d_{H}(x, y) \leq \underline{\lim } d_{H_{i}}\left(x_{i}, y_{i}\right)$;

(2) $d_{H_{i}}\left(x_{i}, y_{i}\right) \rightarrow d_{H}(x, y)$ iff $x_{i} \rightarrow x$ strongly.

Proof. (1): We set $\gamma:=x y$. Take a net $\hat{x}_{i} \in H_{i}$ strongly converging to $x$ and set $\gamma_{i}:=\hat{x}_{i} y_{i}$. It follows from the assumption that $\pi_{\gamma_{i}}\left(x_{i}\right) \rightarrow x$. We have

$$
\underline{\lim } d_{H_{i}}\left(x_{i}, y_{i}\right) \geq \underline{\lim } d_{H_{i}}\left(\pi_{\gamma_{i}}\left(x_{i}\right), y_{i}\right)=d_{H}(x, y) \text {. }
$$

(2): The part of 'if' is obvious. We shall prove the part of 'only if'. Since the equalities in the proof of (1) hold, we have $d_{H_{i}}\left(x_{i}, \pi_{\gamma_{i}}\left(x_{i}\right)\right) \rightarrow 0$ by the triangle comparison. Hence, the limits of $x_{i}$ and $\pi_{\gamma_{i}}\left(x_{i}\right)$ should coincide to each other and to $x$.

Lemma 5.4. Let $x_{i} \in H_{i}$ be a net and $\gamma_{i}, \sigma_{i}:[0,1] \rightarrow H_{i}$ geodesic segments such that

Then we have

$$
\lim _{i} d_{H_{i}}\left(\gamma_{i}(0), \sigma_{i}(0)\right)=\lim _{i} d_{H_{i}}\left(\gamma_{i}(1), \sigma_{i}(1)\right)=0
$$

$$
\lim _{i} d_{H_{i}}\left(\pi_{\gamma_{i}}\left(x_{i}\right), \pi_{\sigma_{i}}\left(x_{i}\right)\right)=0 .
$$

Proof. It follows from the assumption and the convexity of

$$
[0,1] \ni t \mapsto d_{H_{i}}\left(\gamma_{i}(t), \sigma_{i}(t)\right)
$$

(see II.2 of [2]) that

$$
\limsup _{i} \sup _{t} d_{H_{i}}\left(\gamma_{i}(t), \sigma_{i}(t)\right)=0 .
$$

We set $y_{i}:=\pi_{\gamma_{i}}\left(x_{i}\right), z_{i}:=\pi_{\sigma_{i}}\left(x_{i}\right)$, and take $s_{i}, t_{i} \in[0,1]$ as to satisfy $\gamma_{i}\left(s_{i}\right)=y_{i}$ and $\sigma_{i}\left(t_{i}\right)=z_{i}$. (5.1) leads to

$$
\lim _{i} d_{H_{i}}\left(\sigma_{i}\left(s_{i}\right), y_{i}\right)=\lim _{i} d_{H_{i}}\left(\gamma_{i}\left(t_{i}\right), z_{i}\right)=0 .
$$

Since $d_{H_{i}}\left(x_{i}, y_{i}\right) \leq d_{H_{i}}\left(x_{i}, \gamma_{i}\left(t_{i}\right)\right)$ and $d_{H_{i}}\left(x_{i}, z_{i}\right) \leq d_{H_{i}}\left(x_{i}, \sigma_{i}\left(s_{i}\right)\right)$, we have

$$
\begin{aligned}
\lim _{i}\left|d_{H_{i}}\left(x_{i}, y_{i}\right)-d_{H_{i}}\left(x_{i}, z_{i}\right)\right| & =0, \\
\lim _{i}\left|d_{H_{i}}\left(x_{i}, y_{i}\right)-d_{H_{i}}\left(x_{i}, \gamma_{i}\left(t_{i}\right)\right)\right| & =0 .
\end{aligned}
$$


By the triangle comparison,

$$
d_{H_{i}}\left(\gamma_{i}\left(t_{i}\right), y_{i}\right)^{2}+d_{H_{i}}\left(x_{i}, y_{i}\right)^{2} \leq d_{H_{i}}\left(x_{i}, \gamma_{i}\left(t_{i}\right)\right)^{2} .
$$

Therefore, $d_{H_{i}}\left(\gamma_{i}\left(t_{i}\right), y_{i}\right) \rightarrow 0$ and so $d_{H_{i}}\left(y_{i}, z_{i}\right) \rightarrow 0$.

Lemma 5.5. Any bounded sequence $x_{i} \in H_{i}$ has a weakly convergent subnet.

Proof. The proof is based on that by Jost [10]. We may assume that $\left\{x_{i}\right\}$ is a countable sequence, i.e., $i=1,2, \ldots$ Take a dense countable subset $\left\{\xi_{\nu}\right\}_{\nu \in \mathbb{N}} \subset H$. Let $y_{0} \in H$ and $y_{0, i} \in H_{i}$ be such that $y_{0, i} \rightarrow y_{0}$ strongly. For each $\nu \in \mathbb{N}$, we take a sequence $\xi_{\nu, i} \in H_{i}$ converging to $\xi_{\nu}$. Then we have $\gamma_{\nu, i}^{0}:=y_{0, i} \xi_{\nu, i} \rightarrow \gamma_{\nu}^{0}:=$ $y_{0} \xi_{\nu}(i \rightarrow \infty)$. Since $d_{H_{i}}\left(x_{i}, y_{0, i}\right)$ is bounded, $w_{\nu, i}^{0}:=\pi_{\gamma_{\nu, i}^{0}}\left(x_{i}\right)$ has a convergent subsequence whose limit, say $w_{\nu}^{0}$, is a point in $\gamma_{\nu}^{0}$. By a diagonal argument, we can choose a common subsequence of $\{i\}$ independent of $\nu$ for which $w_{\nu, i}^{0}$ converges to $w_{\nu}^{0}$ as $i \rightarrow \infty$ and denote the subsequence by the same notation $\{i\}$. We take a sequence $\epsilon_{m} \rightarrow 0+, m=0,1,2, \ldots$ Let us define $y_{m, i}$ and $y_{m}$ inductively as follows. Suppose that $y_{m, i}$ and $y_{m}$ are defined and satisfy $\lim _{i} y_{m, i}=y_{m}$. We shall define $y_{m+1}$ and $y_{m+1, i}$. By setting $\gamma_{\nu, i}^{m}:=y_{m, i} \xi_{\nu, i}$, the sequence $w_{\nu, i}^{m}:=\pi_{\gamma_{\nu, i}^{m}}\left(x_{i}\right)$ has a convergent subsequence, which can be chosen to be independent of $\nu$ by a diagonal argument. We replace the sequence $\{i\}$ by such a subsequence and set $w_{\nu}^{m}:=\lim _{i} w_{\nu, i}^{m}$. There exists a number $\nu(m+1) \in \mathbb{N}$ such that

$$
d_{H}\left(y_{m}, w_{\nu(m+1)}^{m}\right)>\sup _{\nu \in \mathbb{N}} d_{H}\left(y_{m}, w_{\nu}^{m}\right)-\epsilon_{m} .
$$

Define $y_{m+1}:=w_{\nu(m+1)}^{m}$ and $y_{m+1, i}:=w_{\nu(m+1), i}^{m}$. This defines sequences $y_{m, i}$ and $y_{m}, m, i=1,2, \ldots$, such that $\lim _{i} y_{m, i}=y_{m}$ for each $m$.

By the triangle comparison, we have

$$
d_{H_{i}}\left(y_{m, i}, y_{m+1, i}\right)^{2}+d_{H_{i}}\left(y_{m+1, i}, x_{i}\right)^{2} \leq d_{H_{i}}\left(y_{m, i}, x_{i}\right)^{2} .
$$

Taking a subsequence of $\{i\}$ again, we assume that, for each $m, d_{H_{i}}\left(y_{m, i}, x_{i}\right)$ converges to some number, say $\lambda_{m}$. Since $d_{H}\left(y_{m}, y_{m+1}\right)^{2}+\lambda_{m+1}^{2} \leq \lambda_{m}^{2},\left\{\lambda_{m}^{2}\right\}$ is monotone nonincreasing and $d_{H}\left(y_{m}, y_{m+1}\right) \rightarrow 0$. For any $\nu \in \mathbb{N}$,

$$
\lim _{i} d_{H_{i}}\left(y_{m, i}, w_{\nu, i}^{m}\right)=d_{H}\left(y_{m}, w_{\nu}^{m}\right)<\epsilon_{m}+d_{H}\left(y_{m}, y_{m+1}\right)=: \epsilon_{m}^{\prime} \rightarrow 0 .
$$

There exists $\{\nu(l, i)\}$ such that $\lim _{i} \xi_{\nu(l, i)}=y_{l}$. By (5.2), if $\nu \ll i$, then $d_{H_{i}}\left(y_{m, i}, w_{\nu, i}^{m}\right)$ $\leq \epsilon_{m}^{\prime}$. We may assume that $\nu(l, i) \ll i$. Then we have

$$
\varlimsup_{i} d_{H_{i}}\left(y_{m, i}, w_{\nu(l, i), i}^{m}\right) \leq \epsilon_{m}^{\prime} .
$$

Since $\lim _{i} \gamma_{\nu(l, i), i}^{m}=y_{m} y_{l}$, we can choose a common subsequence $\{i\}$ independent of $m$ and $l$ for which $w_{\nu(l, i), i}^{m} \in \gamma_{\nu(l, i), i}^{m}$ converges to some point on $y_{m} y_{l}$, say $x_{m, l}$. By the above inequality,

$$
d_{H}\left(y_{m}, x_{m, l}\right) \leq \epsilon_{m}^{\prime} .
$$

It follows from Lemma 5.4 that $\pi_{y_{m, i} y_{l, i}}\left(x_{i}\right) \rightarrow x_{m, l}$ as $i \rightarrow \infty$. Since $\pi_{y_{m, i} y_{l, i}}\left(x_{i}\right)=$ $\pi_{y_{l, i} y_{m, i}}\left(x_{i}\right)$ we have $x_{m, l}=x_{l, m}$. Therefore,

$$
d_{H}\left(y_{l}, y_{m}\right) \leq d_{H}\left(y_{l}, x_{l, m}\right)+d_{H}\left(x_{l, m}, y_{m}\right) \leq \epsilon_{l}^{\prime}+\epsilon_{m}^{\prime}
$$

and $\left\{y_{m}\right\}$ is a Cauchy sequence. Let $x:=\lim _{m} y_{m}$. By (5.2), for any geodesic $\gamma$ emanating from $x$, there exists a sequence of geodesic segments $\gamma_{i} \subset H_{i}$ strongly converging to $\gamma$ such that $\pi_{\gamma_{i}}\left(x_{i}\right) \rightarrow x$ strongly. Moreover, it follows from Lemma 5.4 that $x_{i} \rightarrow x$ weakly. This completes the proof. 
Remark 5.6. A weakly convergent net $x_{i} \in H_{i}$ is not necessarily bounded. For example, let $\gamma_{i}, i=1,2, \ldots$, be an infinite sequence of different rays in $\mathbb{R}^{2}$ emanating from the origin $o$, and let $H$ be the subspace of $\mathbb{R}^{2}$ consisting of the union of all $\gamma_{i}$. We assume that $H$ is equipped with its intrinsic (geodesic) metric. Then, $H$ is a $\operatorname{CAT}(0)$-space (actually a tree). The sequence $\gamma_{i}(i)$ is unbounded, but weakly converges to the origin $o$.

5.3. Mosco convergence and resolvent. We give functions $E_{i}: H_{i} \rightarrow[0,+\infty]$ and $E: H \rightarrow[0,+\infty]$.

Definition 5.7 (Mosco convergence). We say that $E_{i}$ converges to $E$ in the Mosco sense if both $(\Gamma 1)$ in Definition 4.4 and the following hold:

$\left(\Gamma 2^{\prime}\right)$ If $H_{i} \ni x_{i} \rightarrow x \in H$ weakly, then $E(x) \leq \underline{\lim } E_{i}\left(x_{i}\right)$.

Note that $\left(\Gamma 2^{\prime}\right)$ is a stronger condition than $(\Gamma 2)$, so that a Mosco convergence implies a $\Gamma$-convergence.

Jost's definition of Mosco convergence in [14] seems unfitting in view of Mosco's original definition. We adopt the original definition of Mosco convergence in Definition 5.7.

It is easy to prove the following proposition. The proof is omitted.

Proposition 5.8. Assume that $\left\{E_{i}\right\}$ is asymptotically compact. Then the following are all equivalent to each other:

(1) $E_{i}$ converges to $E$ in the Mosco sense.

(2) $E_{i} \Gamma$-converges to $E$.

(3) $E_{i}$ compactly converges to $E$.

Definition 5.9 (Moreau-Yosida approximation and resolvent, [11]). For $E: H \rightarrow$ $[0,+\infty]$ we define $E^{\lambda}: H \rightarrow[0,+\infty]$ by

$$
E^{\lambda}(x):=\inf _{y \in H}\left(\lambda E(y)+d_{H}(x, y)^{2}\right), \quad x \in H, \lambda>0
$$

and call it the Moreau-Yosida approximation of $E$. If $E$ is lower semi-continuous and convex and if $E \not \equiv+\infty$, then for any $x \in H$ there exists a unique point, say $J_{\lambda}^{E}(x) \in H$, such that

$$
E^{\lambda}(x)=\lambda E\left(J_{\lambda}^{E}(x)\right)+d_{H}\left(x, J_{\lambda}^{E}(x)\right)^{2} .
$$

This defines a map $J_{\lambda}^{E}: H \rightarrow H$, called the resolvent of $E$.

Note that if $H$ is a Hilbert space and if $E$ is a closed densely defined quadratic form on $H$, then we have $J_{\lambda}^{E}=(I+\lambda A)^{-1}$. Here, $I$ is the identity operator and $A$ the infinitesimal generator associated with $E$, i.e., the self-adjoint operator on $H$ such that $\mathcal{D}(E)=\sqrt{A}$ and $E(x)=(\sqrt{A} x, \sqrt{A} x)_{H}$ for any $x \in \mathcal{D}(E)$, where $(\cdot, \cdot)_{H}$ is the Hilbert inner product on $H$.

Definition 5.10 (Strong convergence of maps). We say that a net of maps $f_{i}$ : $H_{i} \rightarrow H_{i}$ strongly converges to a map $f: H \rightarrow H$ if $f_{i}\left(x_{i}\right)$ strongly converges to $f(x)$ for any strongly convergent net $H_{i} \ni x_{i} \rightarrow x \in H$.

Lemma 5.11. Let $E_{i}: H_{i} \rightarrow[0,+\infty]$ be lower semi-continuous and convex. If $E_{i}$ $\Gamma$-converges to $E$, then $E$ is lower semi-continuous and convex. 
Proof. The lower semi-continuity of $E$ follows from Lemma 4.6. We prove the convexity of $E$. Let $\gamma:[0,1] \rightarrow X$ be a given geodesic. There are two nets $x_{i}, y_{i} \in X_{i}$ converging to $\gamma(0), \gamma(1)$ respectively such that $E_{i}\left(x_{i}\right) \rightarrow E(\gamma(0))$ and $E_{i}\left(y_{i}\right) \rightarrow E(\gamma(1))$. For each $i$ we take a geodesic $\gamma_{i}:[0,1] \rightarrow X_{i}$ joining $x_{i}$ to $y_{i}$. Let $t \in[0,1]$ be any number. The convexity of $E_{i}$ says that

$$
\left.E_{i}\left(\gamma_{i}(t)\right)\right) \leq(1-t) E_{i}\left(\gamma_{i}(0)\right)+t E_{i}\left(\gamma_{i}(1)\right)
$$

Since $\gamma_{i}(t)$ converges to $\gamma(t)$ and by $(\Gamma 2)$, we have

$$
E(\gamma(t)) \leq \varliminf_{i} E_{i}\left(\gamma_{i}(t)\right) \leq(1-t) E(\gamma(0))+t E(\gamma(1)) .
$$

This completes the proof.

In what follows, we assume that functions $E_{i}: H_{i} \rightarrow[0,+\infty]$ and $E: H \rightarrow$ $[0,+\infty]$ are all lower semi-continuous, convex, and are not identically equal to $+\infty$. Let $J_{\lambda}^{i}$ and $J_{\lambda}$ be the resolvents of $E_{i}$ and $E$ respectively.

Proposition 5.12. If $E_{i}$ converges to $E$ in the Mosco sense, then for any $\lambda>0$ we have the following:

(1) $E_{i}^{\lambda}$ strongly converges to $E^{\lambda}$.

(2) $J_{\lambda}^{i}$ strongly converges to $J_{\lambda}$.

Proof. Assume that $E_{i}$ converges to $E$ in the Mosco sense and let $H_{i} \ni x_{i} \rightarrow x \in H$. We set $y_{i}:=J_{\lambda}^{i}\left(x_{i}\right)$ and $y:=J_{\lambda}(x)$. Since $E \not \equiv+\infty$, we have $E^{\lambda}(x)<+\infty$ and hence $E(y)<+\infty$. We shall prove that

$$
\varlimsup_{i} E^{\lambda}\left(x_{i}\right) \leq E^{\lambda}(x)=\lambda E(y)+d_{H}(x, y)^{2}<+\infty .
$$

In fact, by $(\Gamma 2)$, there exists a net $z_{i} \in H_{i}$ such that $z_{i} \rightarrow y$ and $E_{i}\left(z_{i}\right) \rightarrow E(y)$. It follows that $E^{\lambda}\left(x_{i}\right) \leq \lambda E\left(z_{i}\right)+d_{H_{i}}\left(x_{i}, z_{i}\right)^{2}$, the right-hand side of which converges to $\lambda E(y)+d_{H}(x, y)^{2}$. This proves $(5.3)$.

Since $E^{\lambda}\left(x_{i}\right)=\lambda E\left(y_{i}\right)+d_{H_{i}}\left(x_{i}, y_{i}\right)^{2}$, this and (5.3) together imply that $\left\{y_{i}\right\}$ is bounded, so that it has a weakly convergent subnet. We replace $\left\{y_{i}\right\}$ with the weakly convergent subnet and denote its weak limit by $y^{\prime}$. By $\left(\Gamma 2^{\prime}\right)$ and Lemma 5.3 we have

$$
\begin{aligned}
\lambda E(y)+d_{H}(x, y)^{2} & \leq \lambda E\left(y^{\prime}\right)+d_{H}\left(x, y^{\prime}\right)^{2} \\
& \leq \frac{\lim }{i}\left(\lambda E\left(y_{i}\right)+d_{H}\left(x_{i}, y_{i}\right)^{2}\right) .
\end{aligned}
$$

By (5.3), the inequalities above become equalities and so we have $y=y^{\prime}$ and $d_{H_{i}}\left(x_{i}, y_{i}\right) \rightarrow d_{H}(x, y)$. Thus, $y_{i}$ strongly converges to $y$ and $E^{\lambda}\left(x_{i}\right) \rightarrow E^{\lambda}(x)$.

Proposition 5.13. If $E_{i}^{\lambda}$ strongly converges to $E^{\lambda}$ for any $\lambda>0$, then $E_{i} \Gamma$ converges to $E$.

Proof. The proof is essentially same as in Lemma 1.4.3 of [14]. Assume that $E_{i}^{\lambda}$ strongly converges to $E^{\lambda}$ for any $\lambda>0$. Let a net $x_{i} \in H_{i}$ converge to a point $x \in H$. Then we have

$$
\frac{\lim _{i}}{i} E_{i}\left(x_{i}\right) \geq \lim _{i} \frac{1}{\lambda} E_{i}^{\lambda}\left(x_{i}\right)=\frac{1}{\lambda} E^{\lambda}(x) \rightarrow E(x) \quad \text { as } \lambda \rightarrow 0+.
$$


The remainder is to prove the existence of a net $y_{i} \in H_{i}$ such that $y_{i} \rightarrow x$ and $\varlimsup_{i} E_{i}\left(y_{i}\right) \leq E(x)$. We may assume that $E(x)<+\infty$. It follows from the assumption that for any $\lambda>0$,

$$
E(x) \geq \frac{1}{\lambda} E^{\lambda}(x)=\lim _{i} \frac{1}{\lambda} E_{i}^{\lambda}\left(x_{i}\right) .
$$

A diagonal argument shows that there exists a net $\lambda_{i} \rightarrow 0+$ such that if we set $y_{i}:=J_{\lambda_{i}}^{i}\left(x_{i}\right)$, then

$$
E(x) \geq \lim _{i} \frac{1}{\lambda_{i}} E_{i}^{\lambda_{i}}\left(x_{i}\right)=\lim _{i}\left(E_{i}\left(y_{i}\right)+\frac{1}{\lambda_{i}} d_{H_{i}}\left(x_{i}, y_{i}\right)^{2}\right) .
$$

This implies that $E(x) \geq \varlimsup_{i} E_{i}\left(y_{i}\right)$ and $d_{H_{i}}\left(x_{i}, y_{i}\right)^{2} \rightarrow 0$. Thus, $y_{i}$ converges to $x$. This completes the proof.

Remark 5.14. For quadratic forms in Hilbert spaces, the strong convergence $E_{i}^{\lambda} \rightarrow$ $E^{\lambda}$ is equivalent to the Mosco convergence $E_{i} \rightarrow E$ (see Theorem 5.27). We do not know if this still holds in the general case.

Remark 5.15. The converse of Proposition 5.13 does not hold. In fact, let $H$ be a Hilbert space and $\left\{e_{i}\right\}_{i \in \mathbb{N}}$ a complete orthonormal basis on $H$. We define $E_{i}(x):=\left\|x-e_{i}\right\|_{H}^{2}$ and $E(x):=\|x\|_{H}^{2}+1$ for any $x \in H$, where $\|\cdot\|_{H}$ denotes the Hilbert norm. Then, it is easy to see that $E_{i}$ strongly converges to $E$ and in particular, $E_{i} \Gamma$-converges to $E$. However, we have, for any $\lambda>0$,

$$
\begin{aligned}
E_{i}^{\lambda}(o) & =\frac{\lambda}{1+\lambda}, & J_{i}^{\lambda}(o) & =\frac{\lambda}{1+\lambda} e_{i}, \\
E^{\lambda}(o) & =1, & J_{\lambda}(o) & =o .
\end{aligned}
$$

Thus, $E_{i}^{\lambda}$ (resp. $J_{i}^{\lambda}$ ) does not strongly converge to $E^{\lambda}$ (resp. $J_{\lambda}$ ).

Propositions 5.8, 5.12, and 5.13 together imply the following

Corollary 5.16. Assume that $\left\{E_{i}\right\}$ is asymptotically compact. Then, the following are equivalent:

(1) $E_{i}$ compactly converges to $E$.

(2) $E_{i}^{\lambda}$ strongly converges to $E^{\lambda}$ for any $\lambda>0$.

Definition 5.17 (Asymptotic compactness of maps). We say that a net of maps $\left\{f_{i}: H_{i} \rightarrow H_{i}\right\}$ is asymptotically compact if for any bounded net $x_{i} \in H_{i},\left\{f_{i}\left(x_{i}\right)\right\}$ has a strongly convergent subnet. We say that $f_{i}: H_{i} \rightarrow H_{i}$ compactly converges to a map $f: H \rightarrow H$ if $\left\{f_{i}\right\}$ is asymptotically compact and strongly converges to $f$.

Proposition 5.18. Assume that there exists a bounded net $z_{i} \in H_{i}$ such that $\left\{E\left(z_{i}\right)\right\}$ is bounded. Then the following are equivalent:

(1) $\left\{E_{i}\right\}$ is asymptotically compact.

(2) $\left\{J_{\lambda}^{i}\right\}$ is asymptotically compact for any $\lambda>0$.

Proof. (1) $\Longrightarrow(2)$ : Assume (1). Let $x_{i} \in H_{i}$ be a bounded net and $\lambda>0$ be fixed. Then, $E_{i}\left(J_{\lambda}^{i}\left(x_{i}\right)\right)+d_{H_{i}}\left(J_{\lambda}^{i}\left(x_{i}\right), x_{i}\right)^{2} \leq E_{i}\left(z_{i}\right)+d_{H_{i}}\left(x_{i}, z_{i}\right)^{2}$ is bounded. Hence, the asymptotic compactness of $\left\{E_{i}\right\}$ yields that $\left\{J_{\lambda}^{i}\left(x_{i}\right)\right\}$ has a strongly convergent subnet. Thus we obtain (2). 
$(2) \Longrightarrow(1)$ : Assume (2). Let $x_{i} \in H_{i}$ be a bounded net $\operatorname{such}_{\text {that }} \sup _{i} E_{i}\left(x_{i}\right)=$ : $C<+\infty$. To prove (1), it suffices to show the existence of a convergent subnet of $\left\{x_{i}\right\}$. Since $\lambda E\left(J_{\lambda}^{i}\left(x_{i}\right)\right)+d_{H_{i}}\left(J_{\lambda}^{i}\left(x_{i}\right), x_{i}\right)^{2} \leq \lambda E\left(x_{i}\right)$, we have

$$
d_{H_{i}}\left(J_{\lambda}^{i}\left(x_{i}\right), x_{i}\right)^{2} \leq \lambda C \text {. }
$$

By (2), we have a convergent subnet of $J_{\lambda}^{i}\left(x_{i}\right)$ depending on each $\lambda>0$. Take a sequence $\lambda_{k} \rightarrow 0+$. By a diagonal argument, there exists a common subnet of $\left\{x_{i}\right\}$ for which $y_{k, i}:=J_{\lambda_{k}}^{i}\left(x_{i}\right)$ converges for all $k$. Set $y_{k}:=\lim _{i} y_{k, i}$. It follows from (5.4) that $d_{H_{i}}\left(y_{k, i}, y_{l, i}\right) \leq\left(\sqrt{\lambda_{k}}+\sqrt{\lambda_{l}}\right) \sqrt{C}$ and hence $d_{H}\left(y_{k}, y_{l}\right) \leq\left(\sqrt{\lambda_{k}}+\sqrt{\lambda_{l}}\right) \sqrt{C}$, so that $\left\{y_{k}\right\}$ is a Cauchy sequence in $H$. Denote the limit of $\left\{y_{k}\right\}$ by $y$. Again by a diagonal argument, we can choose a subnet $\{i(k)\}$ of $\{i\}$ in such a way that $y_{k, i(k)}$ converges to $y$. Since $d_{H_{i(k)}}\left(y_{k, i(k)}, x_{i(k)}\right) \leq \sqrt{\lambda_{k} C} \rightarrow 0$, the sequence $\left\{x_{i(k)}\right\}$ converges to $y$. This completes the proof.

Remark 5.19. The existence of $\left\{z_{i}\right\}$ in Proposition 5.18 is necessary. In fact, if such a net $\left\{z_{i}\right\}$ does not exist, then (1) is always true, but (2) does not necessarily hold.

Corollary 5.20. The following are equivalent:

(1) $E$ is compact.

(2) $J_{\lambda}$ is compact for any $\lambda>0$, i.e., any bounded subset of $H$ is mapped by $J_{\lambda}$ to a relatively compact set.

Proof. Set $E_{i}=E$ in Proposition 5.18.

Corollary 5.21. The following are equivalent:

(1) $E_{i}$ compactly converges to $E$.

(2) $E_{i}^{\lambda}$ strongly converges to $E^{\lambda}$ and $\left\{J_{\lambda}^{i}\right\}$ is asymptotically compact for any $\lambda>0$.

Proof. (1) $\Longrightarrow$ (2): We assume (1). Let us see the existence of a bounded net $z_{i} \in H_{i}$ such that $\left\{E_{i}\left(z_{i}\right)\right\}$ is bounded. In fact, this follows from the $\Gamma$-convergence of $E_{i}$ to $E$ and $E \not \equiv+\infty$. Thus, Corollary 5.16 and Proposition 5.18 imply (2).

$(2) \Longrightarrow(1)$ : Assume (2). By Corollary 5.16 and Proposition 5.18, it suffices to prove the existence of a bounded net $z_{i} \in H_{i}$ such that $\left\{E_{i}\left(z_{i}\right)\right\}$ is bounded. To see this, we fix a number $\lambda>0$. Since $E \not \equiv+\infty$, we have a point $x \in H$ with $E^{\lambda}(x)<+\infty$. Find a net $x_{i} \in H$ converging to $x$. Then, (2) implies that $E^{\lambda}\left(x_{i}\right) \rightarrow$ $E^{\lambda}(x)<+\infty$. Setting $z_{i}:=J_{\lambda}^{i}\left(x_{i}\right)$, we have $E^{\lambda}\left(x_{i}\right)=\lambda E_{i}\left(z_{i}\right)+d_{H_{i}}\left(x_{i}, z_{i}\right)^{2}$. Therefore, $\left\{z_{i}\right\}$ and $\left\{E_{i}\left(z_{i}\right)\right\}$ are both bounded. This completes the proof.

Theorem 5.22. The following are equivalent:

(1) $E_{i}$ compactly converges to $E+c$ for some constant $c \in \mathbb{R}$.

(2) $J_{\lambda}^{i}$ compactly converges to $J_{\lambda}$ for any $\lambda>0$.

To prove Theorem 5.22, we need the concept of a semigroup, which is studied by Jost and Mayer.

Definition 5.23 (Semigroup, $[25,14]$ ). Let $E: H \rightarrow[0,+\infty]$ be a lower semicontinuous and convex function and $\mathcal{D}(E):=\{x \in H \mid E(x)<+\infty\}$. For any $x \in \overline{\mathcal{D}(E)}$ and $t>0$, there exists the limit

$$
T_{t}^{E}(x):=\lim _{n \rightarrow \infty}\left(J_{t / n}^{E}\right)^{n}(x)
$$

(see $[25,14])$. Define $T_{0}^{E}$ to be the identity map. The family of the maps $T_{t}^{E}: H \rightarrow$ $H, t \geq 0$, is called the semigroup associated with $E$. 
Define

$$
|\nabla(-E)|(x):=\varlimsup_{\substack{y \rightarrow x \\ y \neq x}} \frac{-E(y)+E(x)}{d_{H}(x, y)}, \quad x \in \mathcal{D}(E) .
$$

Theorem 5.24 (Jost-Mayer [25, 14]). (1) For each $t \geq 0, T_{t}^{E}: \overline{\mathcal{D}(E)} \rightarrow H$ is Lipschitz continuous with Lipschitz constant 1 . For each $x \in \overline{\mathcal{D}(E)}$, $t \mapsto T_{t}^{E}(x)$ is continuous on $[0,+\infty)$ and locally Lipschitz continuous on $(0,+\infty)$.

(2) For any $s, t \geq 0$ we have

$$
T_{s+t}^{E}=T_{s}^{E} \circ T_{t}^{E} .
$$

(3) For any fixed $x \in \overline{\mathcal{D}(E)}$, setting $c(t):=T_{t}^{E}(x), t \geq 0$, we have

$$
\begin{aligned}
\lim _{h \rightarrow 0+} \frac{d_{H}(c(t+h), c(t))}{h} & =\lim _{h \rightarrow 0+} \frac{-E(c(t+h))+E(c(t))}{d_{H}(c(t+h), c(t))} \\
& =|\nabla(-E)|(c(t)) .
\end{aligned}
$$

Lemma 5.25. Let $E, F: H \rightarrow[0,+\infty]$ be two lower semi-continuous convex functions with $E, F \not \equiv+\infty$. Then the following are equivalent:

(1) $\mathcal{D}(E)=\mathcal{D}(F)$ and $E-F$ is constant on $\mathcal{D}(E)$.

(2) $J_{\lambda}^{E}=J_{\lambda}^{F}$ for any $\lambda>0$.

(3) $\overline{\mathcal{D}(E)}=\overline{\mathcal{D}(F)}$ and $T_{t}^{E}=T_{t}^{F}$ for any $t \geq 0$.

Proof. The implications $(1) \Longrightarrow(2) \Longrightarrow(3)$ are obvious by the definitions of the resolvent and semigroup.

Let us prove $(3) \Longrightarrow(1)$. Applying Theorem 5.24(3) yields that for any $x \in$ $\overline{\mathcal{D}(E)}$,

$$
E(x)-\inf E=\int_{0}^{+\infty}\left\{|\nabla(-E)|\left(T_{t}^{E}(x)\right)\right\}^{2} d t .
$$

Theorem 5.24(3) implies that $|\nabla(-E)|$ is determined only by the semigroup $T_{t}^{E}$. Since $T_{t}^{E}=T_{t}^{F}$ for any $t \geq 0$, we have $|\nabla(-E)|=|\nabla(-F)|$, so that $E(x)-\inf E=$ $F(x)-\inf F$. This completes the proof.

Proof of Theorem 5.22. (1) $\Longrightarrow$ (2) follows from Proposition 5.12 and Corollary 5.21 .

Let us prove $(2) \Longrightarrow(1)$. Assume (2). Since $\left\{J_{\lambda}^{i}\right\}_{i}$ is asymptotically compact for any $\lambda>0$, Proposition 5.18 implies that $\left\{E_{i}\right\}$ is asymptotically compact and has a compactly convergent subnet. It suffices to show that the limit of any compactly convergent subnet of $\left\{E_{i}\right\}$ coincides with $E$. Take a compactly convergent subnet of $\left\{E_{i}\right\}$ and denote it by the same notation $\left\{E_{i}\right\}$. Let $F: H \rightarrow[0,+\infty]$ be its limit. By Proposition 4.9 and Lemma 5.11, $F$ is compact and convex. By Proposition $5.12, J_{\lambda}^{i}$ strongly converges to $J_{\lambda}^{F}$ for any $\lambda>0$. By (2) we have $J_{\lambda}^{E}=J_{\lambda}^{F}$ for any $\lambda>0$. Applying Lemma 5.25 yields that $\mathcal{D}(E)=\mathcal{D}(F)$ and $E-F$ is constant. This completes the proof.

Problem 5.26. What can we say about the semigroup $T_{t}^{E_{i}}$ of $E_{i}$ associated with the convergence of $E_{i}$ ? See Theorem 5.27 below for the Hilbert case. 
5.4. Case of Hilbert spaces. Throughout this section, we assume that $H$ and $H_{i}$ are real Hilbert spaces that have an asymptotic relation. Let $A$ and $A_{i}$ be selfadjoint operators on $H$ and $H_{i}$ with their spectral measures $\mu$ and $\mu_{i}$ respectively. Denote by $\left\{T_{t}\right\}_{t \geq 0}$ and $\left\{T_{t}^{i}\right\}_{t \geq 0}$ the strongly continuous contraction semigroups $\left(T_{t}:=\right.$ $\left.e^{-t A}, T_{t}^{i}:=e^{-t A_{i}}, t \geq 0\right)$, and by $\left\{J_{\lambda}\right\}_{\lambda>0}$ and $\left\{J_{\lambda}^{i}\right\}_{\lambda>0}$ the strongly continuous resolvents $\left(J_{\lambda}:=(I+\lambda A)^{-1}\right.$ and $\left.J_{\lambda}^{i}:=\left(I+\lambda A_{i}\right)^{-1}, \lambda>0\right)$. We have a densely defined closed quadratic form $\mathcal{E}$ on $H$ defined by $\mathcal{E}(u, v):=(\sqrt{A} u, \sqrt{A} v)_{H}, u, v \in$ $\mathcal{D}(\mathcal{E}):=\mathcal{D}(\sqrt{A})$. We also have $\mathcal{E}_{i}$ in the same manner. We say that a continuous function $\varphi: \mathbb{R} \rightarrow \mathbb{R}$ vanishes at infinity if $\lim _{|x| \rightarrow \infty} f(x)=0$.

By Lemma 3.5(1), we have a compatible, linear metric approximation $\left\{f_{i}\right\}$ for $\left\{H_{i}\right\}$ and $H$ such that $\mathcal{D}\left(f_{i}\right)$ is the subspace consisting of finite linear combinations of a basis of $H$. Therefore, Assumption 2.1 of [22] is satisfied. The strong (weak) topology of Definition 2.4 (2.5) of [22] is compatible with that of this paper. Thus, by the results of Section 2 of [22], we obtain the following:

Theorem 5.27. The following are all equivalent:

(1) $\mathcal{E}_{i} \rightarrow \mathcal{E}$ with respect to the Mosco topology (resp. $\mathcal{E}_{i} \rightarrow \mathcal{E}$ compactly).

(2) $J_{\lambda}^{i} \rightarrow J_{\lambda}$ strongly (resp. compactly) for some $\lambda>0$.

(3) $T_{t}^{i} \rightarrow T_{t}$ strongly (resp. compactly) for some $t>0$.

(4) $\varphi\left(A_{i}\right) \rightarrow \varphi(A)$ strongly (resp. compactly) for any continuous function $\varphi$ : $[0, \infty) \rightarrow \mathbb{R}$ with compact support.

(5) $\varphi_{i}\left(A_{i}\right) \rightarrow \varphi(A)$ strongly (resp. compactly) for any net $\left\{\varphi_{i}:[0, \infty) \rightarrow \mathbb{R}\right\}$ of continuous functions vanishing at infinity which uniformly converges to a continuous function $\varphi:[0, \infty) \rightarrow \mathbb{R}$ vanishing at infinity.

(6) $\mu_{i}((a, b]) \rightarrow \mu((a, b])$ strongly (resp. compactly) for any two real numbers $a<b$ which are not in the point spectrum of $A$.

(7) $\left(\mu_{i} u_{i}, v_{i}\right)_{H_{i}} \rightarrow(\mu u, v)_{H}$ vaguely for any nets $u_{i}, v_{i} \in H_{i}$ and any $u, v \in H$ such that $u_{i} \rightarrow u$ strongly and $v_{i} \rightarrow v$ weakly (resp. $u_{i} \rightarrow u$ weakly and $v_{i} \rightarrow v$ weakly).

Definition 5.28. The strong graph limit $\Gamma_{\infty}$ of $\left\{A_{i}\right\}$ is defined to be the set of pairs $(u, v) \in H \times H$ such that there exists a net of vectors $u_{i} \in \mathcal{D}\left(A_{i}\right)$ with $u_{i} \rightarrow u$ and $A_{i} u_{i} \rightarrow v$ strongly.

Theorem 5.29. The following are all equivalent:

(1) $\mathcal{E}_{i} \rightarrow \mathcal{E}$ with respect to the Mosco topology.

(2) $\varphi_{i}\left(A_{i}\right) \rightarrow \varphi(A)$ strongly for any net $\left\{\varphi_{i}:[0, \infty) \rightarrow \mathbb{R}\right\}$ of bounded continuous functions uniformly converging to a bounded continuous function $\varphi:[0, \infty) \rightarrow \mathbb{R}$.

(3) The strong graph limit $\Gamma_{\infty}$ of $\left\{A_{i}\right\}$ coincides with the graph of $A$.

Proposition 5.30. If $\mathcal{E}_{i} \rightarrow \mathcal{E}$ with respect to the Mosco topology, then

$$
\sigma(A) \subset \lim _{i} \sigma\left(A_{i}\right)
$$

i.e., for any $\lambda \in \sigma(A)$ there exist $\lambda_{i} \in \sigma\left(A_{i}\right)$ tending to $\lambda$.

Define $n(I):=\operatorname{dim} \mu(I) H$ and $n_{i}(I):=\operatorname{dim} \mu_{i}(I) H$ for a Borel subset $I \subset \mathbb{R}$. Note that if $A$ has only discrete spectrum, then $n(I)$ coincides with the number of the eigenvalues in $I$ of $A$ with multiplicities. 
Proposition 5.31. Let $a<b$ be two numbers which are not in the point spectrum of $A$. If $\mathcal{E}_{i} \rightarrow \mathcal{E}$ with respect to the Mosco topology, we have

$$
\frac{\lim _{i}}{i} n_{i}((a, b]) \geq n((a, b]) .
$$

Theorem 5.32. Assume that $\mathcal{E}_{i} \rightarrow \mathcal{E}$ compactly. Then, for any $a, b \in \mathbb{R} \backslash \sigma(A)$ with $a<b$, we have $n_{i}((a, b])=n((a, b])$ for sufficiently large $i$. In particular, the limit set of $\sigma\left(A_{i}\right)$ coincides with $\sigma(A)$.

Remark 5.33. Assume that $\mathcal{E}_{i} \rightarrow \mathcal{E}$ compactly. Then, $\mathcal{E}$ is compact and so $A$ has only discrete spectrum. Even if $\mathcal{E}_{i}$ are not necessarily compact, Theorem 5.32 shows that the bottom of the essential spectrum of $A_{i}$ is divergent to $+\infty$. Thus, for each $k \in \mathbb{N}$, the $k^{t h}$ eigenvalue of $A_{i}$ is well-defined if $i$ is large enough compared with $k$. We shall see an example of asymptotically compact $\left\{E_{i}\right\}$ with noncompact $E_{i}$ in Section 6.1.

Corollary 5.34. Assume that $\mathcal{E}_{i} \rightarrow \mathcal{E}$ compactly. Denote by $\lambda_{k}$ (resp. $\left.\lambda_{k}^{i}\right)$ the $k^{\text {th }}$ eigenvalue of $A$ (resp. $\left.A_{i}\right)$ with multiplicity. ( $\lambda_{k}^{i}$ is defined if $i$ is large enough compared with $k$.) We set $\lambda_{k}:=\infty$ for all $k \geq \operatorname{dim} H+1$ if $\operatorname{dim} H<\infty$, and $\lambda_{k}^{i}:=\infty$ for all $k \geq \operatorname{dim} H_{i}+1$ if $\operatorname{dim} H_{i}<\infty$. Then we have

$$
\lim _{i} \lambda_{k}^{i}=\lambda_{k} \quad \text { for any } k \text {. }
$$

Moreover, let $\left\{\varphi_{k}^{i}\right\}_{k=1,2, \ldots}$ be a (possibly incomplete) orthonormal basis on $H_{i}$ such that $\varphi_{k}^{i}$ is an eigenvector for $\lambda_{k}^{i}$ of $A_{i}$. Then, by replacing it with a sub-directed set of $\{i\}$ if necessarily, for each fixed $k \in \mathbb{N}$ with $k \leq \operatorname{dim} H$, the vector $\varphi_{k}^{i}$ strongly converges to some eigenvector $\varphi_{k}$ for $\lambda_{k}$ of $A$ such that $\left\{\varphi_{k}\right\}_{k=1}^{\operatorname{dim} H}$ is a complete orthonormal basis on $H$.

\section{Application}

6.1. Compact convergence of approximating energy functional. Let $\left(M, d_{M}\right)$ be a compact measured metric space and $\left(Y, d_{Y}\right)$ a proper metric space. Let $p \geq 1$ be a number, $b(x, r)$ a positive function of $x \in M, r>0$, and $h_{\rho}(x, y):=\rho$ or $d_{M}(x, y)$ for $x, y \in M, \rho>0$. For a number $\rho>0$ and a measurable map $u: M \rightarrow Y$, we define the $\rho$-approximating energy density $e_{u}^{\rho}: M \rightarrow[0,+\infty)$ of $u$ by

$$
e_{u}^{\rho}(x):=\frac{1}{b(x, \rho)} \int_{B(x, \rho) \backslash\{x\}}\left(\frac{d_{Y}(u(x), u(y))}{h_{\rho}(x, y)}\right)^{p} d y, \quad x \in M,
$$

and the $\rho$-approximating energy $E^{\rho}(u)$ of $u$ by

$$
E^{\rho}(u):=\frac{1}{2} \int_{M} e_{u}^{\rho}(x) d x \in[0,+\infty]
$$

(see $[10,20,30,23])$. We assume the following two conditions:

(M) There exists a positive function $\Theta(R), R>0$, with $\lim _{R \rightarrow 0} \Theta(R)=1$ such that for any $\rho, R$ with $0<\rho \leq R / 2$, and for any measurable map $u: M \rightarrow Y$, we have

$$
e_{u}^{R} \leq \Theta(R) e_{u}^{\rho} \quad \text { a.e. on } M .
$$

(AR) There exists a constant $\kappa \in(0,1]$ such that

$$
\kappa b(x, r) \leq|B(x, r)| \leq b(x, r)
$$

for any $x \in M$ and $r>0$. 
In $[30,23]$, it is shown that some Bishop and Bishop-Gromov type inequalities (called the measure contraction property) imply the condition (M). In particular, closed Riemannian manifolds with volume measure and compact Alexandrov spaces with Hausdorff measure all satisfy $(\mathrm{M})$ and $(\mathrm{AR})$ for a suitable function $b(x, r)$ (see [23]). It follows from (M) that, as $\rho \rightarrow 0, E^{\rho} \Gamma$-converges to a lower semi-continuous functional $E$ on the set of measurable maps from $M$ to $Y$ with respect to $d_{L^{p}}$ (see $[30,23])$. We call the $\Gamma$-limit $E$ the energy functional. If $Y=\mathbb{R}$, then $E^{\rho}$ and $E$ are both quadratic forms. If $M$ and $Y$ are Riemannian, then, for a suitably chosen $b(x, r), E$ is the usual energy functional defined by their Riemannian metrics up to a constant multiple. If $M$ is Riemannian and if $Y$ is a general metric space, then $E$ is what Korevaar and Schoen studied in [20].

Theorem 6.1. Assume that the 1-local covering order of $M$ is at most $o\left(r^{-p}\right)$ and that $(\mathrm{M})$ and $(\mathrm{AR})$ are both satisfied. Then, the $\rho$-approximating energy functional $E^{\rho}$ compactly converges to the energy functional $E$ as $\rho \rightarrow 0$ with respect to $d_{L^{p}}$.

Proof. Since $E^{\rho} \Gamma$-converges to $E$, it suffices to prove that $\left\{E^{\rho}\right\}$ is asymptotically compact. For any $r$ and $\rho$ with $0<\rho \leq r$, we have

$$
\begin{aligned}
& \frac{1}{B(x, r)} \iint_{B(x, r) \times B(x, r)} d_{Y}(u(y), u(z))^{p} d z d y \\
& \leq \frac{1}{B(x, r)} \int_{B(x, r)} \int_{B(y, 2 r)} d_{Y}(u(y), u(z))^{p} d z d y \\
& \leq \frac{2 b(x, r)(2 r)^{p}}{|B(x, r)|} \int_{B(x, r)} e_{u}^{2 r}(y) d y \\
& \leq 2^{p+1} \kappa^{-1} \Theta(2 r) r^{p} \int_{B(x, r)} e_{u}^{\rho}(y) d y,
\end{aligned}
$$

which verifies the Poincaré inequality $(P)_{p, 1, C, \rho, R}$ for some $C$ and $R$. Applying Theorem 4.15 yields the asymptotic compactness of $\left\{E^{\rho}\right\}$. This completes the proof.

Theorem 6.1 and Proposition 4.9 imply the following:

Corollary 6.2. $E$ is compact with respect to $d_{L^{p}}$.

Theorem 6.1 and Proposition 4.11(2) imply:

Corollary 6.3. Let $\rho_{i} \rightarrow 0$ be a sequence of positive numbers and let a net of measurable maps $u_{i}: M \rightarrow Y$ be a $d_{L^{p}}$-bounded asymptotic minimizer of $\left\{E^{\rho_{i}}\right\}$ (see Definition 4.10). Then, $\left\{u_{i}\right\}$ has a subsequence $L^{p}$-converging to a minimizer of $E$.

The following proposition says that $E^{\rho}$ is noncompact typically, although $\left\{E^{\rho}\right\}$ is asymptotically compact.

Proposition 6.4. Assume that $h_{\rho}(x, y):=\rho$ and let $y_{0} \in Y$ be a point. Then, $E^{\rho}$ is compact for a number $\rho>0$ iff $L_{y_{0}}^{p}(M, Y)$ is proper.

Proof. If $L_{y_{0}}^{p}(M, Y)$ is proper, the compactness of $E^{\rho}$ is trivial.

Let us prove the converse. Take any measurable map $u: M \rightarrow Y$. Since

$$
d_{Y}(u(x), u(y))^{p} \leq 2^{p-1}\left(d_{Y}\left(u(x), y_{0}\right)^{p}+d_{Y}\left(u(y), y_{0}\right)^{p}\right),
$$


we have

$$
\begin{aligned}
E^{\rho}(u) & =\frac{1}{2} \iint_{0<d_{M}(x, y)<\rho} \frac{1}{b(x, \rho)} \frac{d_{Y}(u(x), u(y))^{p}}{\rho^{p}} d x d y \\
& \leq \frac{2^{p-1}}{2 \rho^{p} \inf _{x \in M} b(x, \rho)} \iint_{M \times M}\left(d_{Y}\left(u(x), y_{0}\right)^{p}+d_{Y}\left(u(y), y_{0}\right)^{p}\right) d x d y \\
& \leq \frac{2^{p-1}|M|}{\rho^{p} \inf _{x \in M} b(x, \rho)} d_{L^{p}}\left(u, y_{0}\right)^{p} .
\end{aligned}
$$

It follows from $(\mathrm{AR})$ that $\inf _{x \in M} b(x, \rho)>0$. Assume that $E^{\rho}$ is compact. Take an $L^{p}$-bounded net $u_{i} \in L_{y_{0}}^{p}(M, Y)$. Then, by $(6.1), E^{\rho}\left(u_{i}\right)$ is bounded. The compactness of $E^{\rho}$ tells us that $\left\{u_{i}\right\}$ has an $L^{p}$-convergent subnet. This completes the proof.

We now assume that $p=2$ and $Y=\mathbb{R}$, so that $E^{\rho}$ and $E$ are densely defined, nonnegative, and quadratic forms on $L^{2}(M)$. Proposition 6.4 and Theorems 6.1, 5.32 imply:

Corollary 6.5. The spectrum for $E$ is discrete (with only finite multiplicities). Whenever $L^{2}(M)$ has infinite dimension, each $E^{\rho}$ is noncompact and has nonempty essential spectrum. As $\rho \rightarrow 0+$, the bottom of the essential spectrum for $E^{\rho}$ is divergent to $+\infty$, and for any fixed $k \in \mathbb{N}$, the $k^{\text {th }}$ eigenvalue for $E^{\rho}$ converges to that for $E$. The eigenfunctions for $E^{\rho}$ also converge to eigenfunctions for $E$ in the sense of Corollary 5.34.

6.2. Compactness and convergence with a lower bound of Ricci curvature. For constants $n \geq 2$ and $D>0$, let $\mathcal{M}$ be the family of $n$-dimensional closed Riemannian manifolds of Ricci curvature $\geq-(n-1)$ and diameter $\leq D$. We employ the probability measure induced from the Riemannian volume measure on each $M \in \mathcal{M}$. The Gromov compactness theorem shows that the measured GromovHausdorff closure of $\mathcal{M}$ is compact (see (2.11) of [7]). Let $\mathcal{Y}$ be a Gromov-Hausdorff compact family of proper pointed metric spaces. For $M \in \mathcal{M}$ and $(Y, y) \in \mathcal{Y}$, we denote by $E_{M, Y}: L_{y}^{2}(M, Y) \rightarrow[0,+\infty]$ the Korevaar-Schoen type energy (see [20]). Consider the family $E_{\mathcal{M}, \mathcal{Y}}$ of $E_{M, Y}$ for all $M \in \mathcal{M}$ and $Y \in \mathcal{Y}$. We have the following:

Theorem 6.6. Any net of $E_{\mathcal{M}, \mathcal{Y}}$ is asymptotically compact and has a compactly convergent subnet.

Proof. By a result of Buser [3] we have a uniform bound of Poincaré constants, namely $(P)_{2, c, C, 0, R}$ in Section 4.2 holds for $Y=\mathbb{R}$ and $E_{M, Y} \in E_{\mathcal{M}, \mathcal{Y}}$. Moreover, we have a uniform doubling constant by the Bishop-Gromov volume comparison theorem, which implies that any measured Gromov-Hausdorff limit of $\mathcal{M}$ satisfies the doubling condition. According to [21], $(P)_{2, c, C, 0, R}$ for $Y=\mathbb{R}$ implies $(P)_{2, c, C, 0, R}$ for general $Y$. Thus, the assumption of Theorem 4.15 is satisfied for any net of functionals in $E_{\mathcal{M}, \mathcal{Y}}$. This completes the proof.

Consequently, by Theorem 4.25, the family of $\left(\left\{u \in L_{y}^{2}(M, Y) \mid E_{M, Y}(u) \leq\right.\right.$ $c\}, y), M \in \mathcal{M},(Y, y) \in \mathcal{Y}$, is relatively compact with respect to the pointed Gromov-Hausdorff topology. Let $\mathcal{H}$ be a Gromov-Hausdorff compact family of proper pointed CAT(0)-spaces. Then, Proposition 5.18 implies that for any net $\left\{E_{i}\right\}$ in $E_{\mathcal{M}, \mathcal{H}}$, the net of the resolvents $J_{\lambda}^{i}$ of $E_{i}$ is asymptotically compact. By 
Theorem 5.22, $J_{\lambda}^{i}$ has a compactly convergent subnet. Also, by Propositions 5.8, 5.12 , and Theorem 4.7, the Moreau-Yosida approximation $E_{i}^{\lambda}$ of $E_{i}$ has a strongly convergent subnet.

Combining Theorem 6.6 and Kasue's result (Theorem 5.1 of [16]) together with the work of Cheeger and Colding [5] therefore implies the following.

Corollary 6.7. Assume that $(Y, y)$ is a pointed complete Riemannian manifold. Let $M_{i} \in \mathcal{M}$ be a net converging to a measured metric space with respect to the measured Gromov-Hausdorff topology. Then, $E_{M_{i}, Y}$ compactly converges to the energy functional defined in $[15,16]$.

Relative to this corollary, we refer to Theorem 5.4 and Remark 5.1 of [22] for the case $Y=\mathbb{R}$.

Conjecture 6.8. Assume that a net $M_{i} \in \mathcal{M}$ measured Gromov-Hausdorff converges to a measured metric space $M$ and that a net $\left(Y_{i}, y_{i}\right) \in \mathcal{Y}$ pointed GromovHausdorff converges to a proper metric space $(Y, y)$. The limit of $E_{M_{i}, Y_{i}}$ could be uniquely determined only by $M$ and $(Y, y)$.

\section{REFERENCES}

1. S. G. Bobkov, Isoperimetric and analytic inequalities for log-concave probability measures, Ann. Probab. 27 (1999), no. 4, 1903-1921. MR1742893 (2001h:60026)

2. M. R. Bridson and A. Haefliger, Metric spaces of non-positive curvature, Grundlehren der Mathematischen Wissenschaften [Fundamental Principles of Mathematical Sciences], vol. 319, Springer-Verlag, Berlin, 1999. MR1744486 (2000k:53038)

3. P. Buser, A note on the isoperimetric constant, Ann. Sci. École Norm. Sup. (4) 15 (1982), no. 2, 213-230. MR0683635 (84e:58076)

4. J. Cheeger, Differentiability of Lipschitz functions on metric measure spaces, Geom. Funct. Anal. 9 (1999), no. 3, 428-517. MR1708448 (2000g:53043)

5. J. Cheeger and T. H. Colding, On the structure of spaces with Ricci curvature bounded below. III, J. Differential Geom. 54 (2000), no. 1, 37-74. MR1815411 (2003a:53044)

6. G. Dal Maso, An introduction to $\Gamma$-convergence, Birkhäuser Boston Inc., Boston, MA, 1993. MR1201152 (94a:49001)

7. K. Fukaya, Collapsing of Riemannian manifolds and eigenvalues of Laplace operator, Invent. Math. 87 (1987), 517-547. MR0874035 (88d:58125)

8. M. Gromov, Metric structures for Riemannian and non-Riemannian spaces, Birkhäuser Boston Inc., Boston, MA, 1999, Based on the 1981 French original, With appendices by M. Katz, P. Pansu and S. Semmes, Translated from the French by Sean Michael Bates. MR1699320 (2000d:53065)

9. P. Hajłasz and P. Koskela, Sobolev met Poincaré, Mem. Amer. Math. Soc. 145 (2000), no. 688, $\mathrm{x}+101$. MR1683160 (2000j:46063)

10. J. Jost, Equilibrium maps between metric spaces, Calc. Var. Partial Differential Equations 2 (1994), no. 2, 173-204. MR1385525 (98a:58049)

11. _ Convex functionals and generalized harmonic maps into spaces of nonpositive curvature, Comment. Math. Helv. 70 (1995), no. 4, 659-673. MR1360608 (96j:58043)

12. Generalized harmonic maps between metric spaces, Geometric analysis and the calculus of variations, Internat. Press, Cambridge, MA, 1996, pp. 143-174. MR1449406 (99a:58045)

13. _ Nonpositive curvature: geometric and analytic aspects, Birkhäuser Verlag, Basel, 1997. MR1451625 (98g:53070)

14. _ Nonlinear Dirichlet forms, New directions in Dirichlet forms, Amer. Math. Soc., Providence, RI, 1998, pp. 1-47.

15. A. Kasue, Convergence of Riemannian manifolds and harmonic maps, preprint, 2000.

16. _ Convergence of Riemannian manifolds and Laplace operators. II, Potential Anal. 24 (2006), no. 2, 137-194. MR2217418 (2006m: 53060)

17. Convergence of metric graphs and energy forms, preprint, 2004. 
18. A. V. Kolesnikov, Convergence of infinite-dimensional Dirichlet forms with changing speed measures, J. Funct. Anal. 230 (2006), no. 2, 382-418. MR2186217 (2006g:31007)

19. Convergence of Dirichlet forms with changing speed measures on $\mathbb{R}^{d}$, Forum Math. 17 (2005), no. 2, 225-259. MR2130907 (2005m:31020)

20. N. J. Korevaar and R. M. Schoen, Sobolev spaces and harmonic maps for metric space targets, Comm. Anal. Geom. 1 (1993), no. 3-4, 561-659. MR1266480 (95b:58043)

21. P. Koskela, N. Shanmugalingam, and J. T. Tyson, Dirichlet forms, Poincaré inequalities, and the Sobolev spaces of Korevaar and Schoen, Potential Anal. 21 (2004), no. 3, 241-262. MR2075670 (2005f:31015)

22. K. Kuwae and T. Shioya, Convergence of spectral structures: a functional analytic theory and its applications to spectral geometry, Comm. Anal. Geom. 11 (2003), no. 4, 599-673. MR2015170 (2004k:53056)

23. - Sobolev and Dirichlet spaces over maps between metric spaces, J. Reine Angew. Math. 555 (2003), 39-75. MR1956594 (2004g:58009)

24. Z. M. Ma and M. Röckner, Introduction to the theory of (nonsymmetric) Dirichlet forms, Universitext, Springer-Verlag, Berlin, 1992. MR1106853 (92g:03001)

25. U. F. Mayer, Gradient flows on nonpositively curved metric spaces and harmonic maps, Comm. Anal. Geom. 6 (1998), no. 2, 199-253. MR1651416 (99m:58067)

26. R. E. Megginson, An introduction to Banach space theory, Graduate Texts in Mathematics, vol. 183, Springer-Verlag, New York, 1998.

27. U. Mosco, Composite media and asymptotic Dirichlet forms, J. Funct. Anal. 123 (1994), no. 2, 368-421. MR1283033 (95d:47088)

28. Y. Ogura, T. Shioya, and M. Tomisaki, Collapsing of warped product spaces and onedimensional diffusion processes, in preparation.

29. S. Ohta, Cheeger type Sobolev spaces for metric space targets, Potential Anal. 20 (2004), 149-175. MR2032946 (2005h:58017)

30. K.-T. Sturm, Diffusion processes and heat kernels on metric spaces, Ann. Probab. 26 (1998), no. 1, 1-55. MR1617040 (99b:31008)

31. C. Vernicos, The macroscopic sound of tori, Pacific J. Math. 213 (2004), no. 1, 121-156. MR2040254 (2005e:58056)

Department of Mathematics, Faculty of Education, Kumamoto University, KumaMOTO, 860-8555, JAPAN

E-mail address: kuwae@gpo.kumamoto-u.ac.jp

Mathematical Institute, Tohoku University, Sendai 980-8578, JAPAN

E-mail address: shioya@math.tohoku.ac.jp 